\title{
Rotation, pulsations and magnetic field in V 2052 Ophiuchi: A new He-strong star $\star, \star \star$
}

\author{
C. Neiner ${ }^{1,2,3}$, H. F. Henrichs ${ }^{1}$, M. Floquet ${ }^{2}$, Y. Frémat ${ }^{2}$, O. Preuss ${ }^{4}$, A.-M. Hubert ${ }^{2}$, V. C. Geers ${ }^{1}$, \\ A. H. Tijani ${ }^{1}$, J. S. Nichols ${ }^{5}$, and S. Jankov ${ }^{6,7}$ \\ 1 Sterrenkundig Instituut “Anton Pannekoek”, Universiteit van Amsterdam, The Netherlands \\ 2 GEPI/UMR 8111 du CNRS, Observatoire de Paris-Meudon, France \\ 3 Current affiliation: RSSD, Estec / ESA, Keplerlaan 1, 2201 AZ, Noordwijk ZH, The Netherlands \\ ${ }^{4}$ Max Planck Institut für Aeronomie, Lindau, Germany \\ ${ }^{5}$ Harvard/Smithsonian Center for Astrophysics, Cambridge, MA, USA \\ ${ }^{6}$ Observatoire de la Côte d'Azur, France \\ 7 Astronomical Observatory Beograd, Yugoslavia
}

Received 13 August 2002 / Accepted 19 August 2003

\begin{abstract}
V $2052 \mathrm{Oph}$ is a $\beta$ Cep star with $v \sin i \sim 60 \mathrm{~km} \mathrm{~s}^{-1}$. The behavior of its stellar wind was studied in the ultraviolet wavelength region with the IUE satellite. It revealed periodic variations in the equivalent widths $(E W)$ of the resonance lines of wind-sensitive ions with a period of $3.638833 \mathrm{~d}$, which is identified as the rotational period. These variations are typical of magnetic stars. Therefore time-resolved circular spectropolarimetric observations were obtained with the Musicos échelle spectropolarimeter at the 2-m Télescope Bernard Lyot (TBL) to search for a magnetic field in the star. Stokes V patterns were observed, the inclination and magnetic angles were derived and a value was determined for the polar magnetic field $(250 \pm 190 \mathrm{G})$ using an oblique rotator dipole model. The spectroscopic information was used to search for periodicity in line-profile variations (lpv), radial velocity and minimum intensity curves. Multiperiodicity was found, corresponding to radial $\left(f_{1}=7.15 \mathrm{c} \mathrm{d}^{-1}\right)$ and non-radial $\left(f_{2}=6.82 \mathrm{c} \mathrm{d}^{-1}, l=3\right.$ or 4$)$ pulsation modes. The rotation period is also detected in rotationally modulated observables because of the magnetic poles passing through the observer's line of sight. We determined the stellar parameters of the star, which was found to be chemically peculiar, in particular He-enriched. This makes V 2052 Oph the first magnetic He-strong early B star with known pulsational properties.
\end{abstract}

Key words. stars: magnetic fields - stars: winds, outflows - stars: chemically peculiar - stars: early-type - stars: oscillations

\section{Introduction}

The B2 IV-V star V 2052 Oph (HD 163472, HR 6684, $V=$ $5.85)$ is classified as a $\beta$ Cep variable. It has one of the shortest periods and lowest luminosity of its type. The period of $3 \mathrm{~h} 21 \mathrm{~min}$ in its light variability discovered by Jerzykiewicz (1972) has also been detected in radial velocity variations (Pike 1974). The periodic variability in the light curve is also reported with high accuracy in the Hipparcos catalogue (Perryman et al. 1997). Morton \& Hansen (1974) deduced temperature variations up to $900 \mathrm{~K}$ from near-UV light variations. Since then, the pulsation period of V 2052 Oph has been well

Send offprint requests to: $\mathrm{C}$. Neiner,

e-mail: cneiner@rssd.esa.int

* Based on observations obtained with the MuSiCoS spectropolarimeter at the Observatoire du Pic du Midi, France, and by the International Ultraviolet Explorer, collected at NASA Goddard Space Flight Center and Villafranca Satellite Tracking Station of the European Space Agency, retrieved from the INES database.

$\star \star$ Table 7 is only available in electronic form at http://www. edpsciences.org studied. Cugier et al. (1994) and Heynderickx et al. (1994) found that it corresponds to a $l=0$ single radial mode.

Rountree \& Sonneborn (1991) classified the star from the photospheric lines in the UV spectrum as B2 IVw, where the w-designation signifies anomalous wind lines. They also noted that the single spectrum they considered looked very similar to that of $\zeta$ Cas, a star being known for its variable C IV wind lines (Sonneborn et al. 1987), and which was recently found to be magnetic (Neiner et al. 2003).

Inspection of the three available spectra in the microfiche atlas of all high-resolution spectra of OB stars taken during the first 10 years of IUE (Bohlin et al. 1994) showed clearly variable wind lines, similar to what is observed in known magnetic B stars, i.e. showing periodic absorption modulations, but very much unlike the variability found in $\mathrm{O}$ stars, i.e. discrete absorption components (DACs). This specific type of wind variations appeared a reliable signature of the presence of a weak stellar magnetic field, such as in the case of $\beta$ Cep (Henrichs et al. 2003), and enables an accurate determination of the rotational period. Henrichs et al. (1998) estimated a 
3.75 day period and predicted a minimum field of a few hundred Gauss.

In addition, studying the pulsation properties of a rotating magnetic star gives strong constraints on its stellar parameters and its evolutionary stage, which is of high asteroseismological importance.

These considerations motivated us to undertake a detailed time-resolved UV study of V 2052 Oph which was intensively monitored with the IUE satellite (March 1994August 1995), to attempt to measure the expected magnetic field (June 2000 and 2001) with the Musicos spectropolarimeter at TBL (Pic du Midi, France), and to analyze the pulsational properties of the star.

In Sect. 2 we present the UV and spectropolarimetric observations. We analyze the UV stellar wind changes and extract the rotational period of V 2052 Oph in Sect. 3. We determine an improved value for the $v \sin i$ of the star, review its stellar parameters and determine its chemical composition in Sect. 4. In Sect. 5 we study the pulsations in the line profiles and radial velocities. In Sect. 6 we report on the measurements of the longitudinal component of the magnetic field, in the context of an oblique magnetic dipole. We discuss the results and present conclusions in Sect. 7.

\section{Observations}

\subsection{Ultraviolet spectroscopy}

High-dispersion ultraviolet spectra $(R \simeq 18000)$ were obtained with the Short Wavelength Prime (SWP) camera onboard the IUE satellite. Table 1 presents the journal of the 41 obtained spectra of V 2052 Oph. 3 spectra taken during 1981 and 1982 were extracted from the IUE archive, whereas 38 spectra were taken in 1994 and during 11-31 August 1995 by one of us (JN). All reduced data were retrieved from the INES database for homogeneity. The spectra were mapped on a uniform wavelength grid of $0.1 \AA$, which effectively degraded the resolving power to $12000-15000$. The signal-to-noise ratio of the spectra is about 25 (see Henrichs et al. 1994).

\subsection{Spectropolarimetry}

The fiber-fed Musicos échelle spectropolarimeter $(R \simeq 35000)$ is mounted at the Cassegrain focus of the 2-m Télescope Bernard Lyot (TBL) at Pic du Midi in France (see Donati et al. 1999). Stellar light is collected in a $2^{\prime \prime}$ entrance aperture in the spectral range 4500-6600 А. Linear/circular sheet polarisers can be inserted in the beam. One half-wave and one quarterwave retarder can also be inserted and rotated to achieve a linear or circular analysis of the stellar light.

To detect stellar magnetic fields, one analyses the circular polarisation of the light. The light is split into two beams, fed to the spectrograph through a double optical fiber and simultaneously recorded onto the $1024 \times 102424 \mu \mathrm{m}$ square pixel SITe CCD detector. The observing strategy is to set the quarterwave plate and take 4 subexposures: one at azimuth $-45^{\circ}$, two at azimuth $45^{\circ}$, and one more at azimuth $-45^{\circ}$. In principle only 2 exposures are needed (one at each azimuth) but with
Table 1. Journal of IUE observations of V 2052 Oph (programs PHCAL and BEPJN). Column 1 indicates the number in the IUE archives. Column 2 gives the exposure time in seconds. The Heliocentric Julian Date (HJD) at mid-exposure minus 2440000 is given in the last column.

\begin{tabular}{llllll}
\hline \hline Image & Exp & Mid & Image & Exp & Mid \\
SWP & s & HJD & SWP & s & HJD \\
\hline 14514 & 1200 & 4804.43 & 55601 & 660 & 9948.19 \\
14515 & 720 & 4804.47 & 55610 & 660 & 9950.12 \\
18313 & 660 & 5260.36 & 55633 & 660 & 9951.19 \\
50411 & 600 & 9440.48 & 55648 & 660 & 9951.85 \\
50431 & 600 & 9442.54 & 55654 & 660 & 9952.10 \\
50636 & 630 & 9470.40 & 55668 & 660 & 9953.03 \\
50639 & 630 & 9470.53 & 55672 & 660 & 9953.19 \\
50642 & 660 & 9470.86 & 55683 & 660 & 9954.04 \\
50644 & 630 & 9471.27 & 55688 & 480 & 9954.20 \\
50651 & 660 & 9471.86 & 55698 & 660 & 9955.06 \\
50658 & 690 & 9472.84 & 55702 & 660 & 9955.19 \\
50659 & 630 & 9473.22 & 55711 & 660 & 9956.03 \\
52122 & 660 & 9611.00 & 55714 & 660 & 9956.16 \\
55480 & 600 & 9941.06 & 55732 & 660 & 9956.90 \\
55488 & 660 & 9942.16 & 55737 & 660 & 9957.11 \\
55506 & 660 & 9943.11 & 55746 & 660 & 9958.15 \\
55526 & 660 & 9944.07 & 55753 & 660 & 9958.99 \\
55531 & 660 & 9944.20 & 55757 & 660 & 9959.12 \\
55535 & 660 & 9944.92 & 55782 & 660 & 9961.07 \\
55540 & 660 & 9945.14 & 55783 & 660 & 9961.10 \\
55571 & 660 & 9946.93 & & & \\
\hline & & & & &
\end{tabular}

4 exposures the path of the two beams are mutually exchanged through the instrument during one complete Stokes V measurement. With this procedure, systematic spurious circular polarisation signals are removed down to an accuracy of $0.002 \%$.

25 Stokes V measurements were obtained in June-July 2000 (observers CN, HH, AT) and 88 Stokes V measurements in June-July 2001 (observers CN, HH, VG), i.e. 113 measurements in total (see Table 2). The individual subexposures can also be used as normal spectroscopic data, including subexposures which do not form a complete set for a magnetic measurement. Therefore we have 518 individual spectra available for pulsation analysis. Their average $S / N$ in the intensity continuum is about 120 .

A dedicated software package, ESpRIT (see Donati et al. 1997), is available at TBL to reduce and analyze the data. We implemented an improved version of ESpRIT, using two series of flat-fields taken in the two positions of the quarterwave plate, which optimizes the extraction of the échelle orders. Fringes were present in the spectra taken in 2001. They were removed using a fringe template extracted from the Stokes V spectrum of a non-magnetic star ( $\zeta \mathrm{Oph})$ taken and reduced in the same way as V $2052 \mathrm{Oph}$. Fringes are probably also present in the data taken in 2000 , but due to the lower quality of these data they could not be identified. Tests on the 
Table 2. Journal of TBL observations of V 2052 Oph obtained in 2000 and 2001. Column 1 indicates the number of the polarimetric set. Columns 2 and 3 show the date and time of the beginning of observations. Column 4 indicates the number of obtained polarimetric sets or individual subexposures. Column 5 gives the exposure time in seconds of each individual subexposure.

\begin{tabular}{|c|c|c|c|c|}
\hline$\#$ & Date & $\begin{array}{l}\text { Start UT } \\
\text { h:min }\end{array}$ & $\begin{array}{l}\text { Sets/ } \\
\text { ind. }\end{array}$ & $\begin{array}{l}\text { Exp } \\
\mathrm{s}\end{array}$ \\
\hline 1 & 2000 Jun. 29 & $20: 39$ & 1 & 2400 \\
\hline 2 & 29 & $23: 49$ & 1 & 2100 \\
\hline $3-7$ & 2000 Jul. 4 & $20: 59$ & 5 & 720 \\
\hline $8-13$ & 5 & $20: 54$ & 6 & 720 \\
\hline $14-16$ & 9 & $20: 31$ & 3 & 720 \\
\hline $17-20$ & 12 & $22: 46$ & 4 & 720 \\
\hline 21 & 16 & $20: 32$ & 1 & 720 \\
\hline $22-25$ & 16 & $21: 58$ & 4 & 720 \\
\hline $26-31$ & 2001 Jun. 14 & $22: 12$ & 6 & 420 \\
\hline $32-33$ & 15 & $24: 07$ & 2 & 420 \\
\hline $34-36$ & 17 & $25: 54$ & 3 & 420 \\
\hline $37-41$ & 19 & 23:06 & 5 & 420 \\
\hline $42-48$ & 20 & $22: 08$ & 7 & 420 \\
\hline $49-54$ & 21 & $21: 53$ & 6 & 420 \\
\hline $55-59$ & 22 & $20: 44$ & 5 & 420 \\
\hline $60-61$ & 22 & $23: 47$ & 2 & 420 \\
\hline $62-64$ & 23 & $20: 30$ & 3 & 420 \\
\hline $65-68$ & 23 & $22: 42$ & 4 & 420 \\
\hline $69-74$ & 24 & $20: 56$ & 6 & 420 \\
\hline $75-81$ & 25 & $20: 48$ & 7 & 420 \\
\hline $82-88$ & 28 & $20: 33$ & 7 & 420 \\
\hline $89-95$ & 29 & $20: 22$ & 7 & 420 \\
\hline $96-101$ & 30 & $20: 29$ & 6 & 420 \\
\hline $102-108$ & 2001 Jul. 1 & $20: 29$ & 7 & 420 \\
\hline \multirow[t]{11}{*}{$109-113$} & 2 & $21: 43$ & 5 & 420 \\
\hline & 2000 Jun. 30 & $21: 20$ & 1 & 1500 \\
\hline & 2000 Jul. 9 & $23: 13$ & 5 & 720 \\
\hline & 12 & $21: 54$ & 3 & 720 \\
\hline & 16 & $21: 40$ & 1 & 720 \\
\hline & 2001 Jun. 14 & $25: 47$ & 1 & 420 \\
\hline & 18 & $21: 43$ & 36 & 420 \\
\hline & 19 & 21:03 & 9 & 420 \\
\hline & 20 & $21: 41$ & 3 & 420 \\
\hline & 22 & $23: 25$ & 3 & 420 \\
\hline & 23 & $22: 06$ & 2 & 420 \\
\hline
\end{tabular}

2001 data showed, however, that when the $S / N$ ratio is low, application of the fringe correction makes no difference in the value derived for the magnetic field.

After applying the Least-Squares Deconvolution (LSD), a cross-correlation technique developed by Donati et al. (1997), one can detect a stellar magnetic field through the Zeeman signatures generated in the shape and polarization state of spectral line profiles. The LSD method combines the very small circularly polarized signatures, properly weighted, of all available line profiles in the spectrum to increase the signal to noise ratio. For V 2052 Oph, a line template of 159 spectral lines was created and used to provide a mean Stokes $\mathrm{V}$ profile. These photospheric lines correspond to ions of $\mathrm{He}$ I, C II, C III, N II, O II, Ne I, Mg II, Al III, Si II, Si III, S II, Ar II, Fe III, with line depths from 0.01 to 0.46 and Landé factors from 0.5 to 2.7. The line depths and Landé factors are extracted from Kurucz models provided in the ESpRIT Package. The method assumes that the intrinsic broadening is similar for all lines. When a magnetic field is present, the Stokes V profile indicates a Zeeman signature.

\subsection{VLT/UVES spectrum}

One single VLT/UVES spectrum was obtained on September 25th 2001 (courtesy of L. Kaper and his colleagues). The resolution is about $R \simeq 100000$ with a signal to noise ratio of $S / N \simeq 850$. The spectrum covers three wavelength domains: [3250-4500], [4580-5555] and [5600-6580] $\AA$. The reduction was not performed with the dedicated UVES pipeline in MIDAS ${ }^{1}$, as this pipeline is optimized for spectra with $S / N<100$. The usual bias and flat-field correction, order extraction and wavelength calibration was done with the IRAF ${ }^{2}$ software package.

\section{UV stellar wind variations}

Figure 1 shows a mean spectrum of 40 (out of 41) available high-resolution IUE spectra (top panels), along with the ratio of the measured to expected variability (lower panels). A ratio higher than unity signals statistically significant variability. Image SWP 14514 was omitted in the average spectrum due to overexposure especially at longer wavelengths. The expected variability was obtained using a noise model for such IUE spectra $S / N=A \times \tanh (F / B)$ as derived by Henrichs et al. (1994), where $F$ is the flux, $A=26$ and $B=1.33 \times 10^{-10}$. The windsensitive doublet resonance lines of C IV 1550, Si IV 1400, N V 1240 and Al III 1860 are strongly variable and are analysed for periodicity below. The highly instrumentally contaminated Si III 1206 line and probably the C III complex at $1175 \AA$ are also variable, but are not considered further.

The top panels of Fig. 2 show an overplot of the IUE C IV, Si IV, N V and Al III line profiles, respectively, along with their variability signatures in the bottom panels. The variability occurs mainly in the strength of the line over a given range in velocity space, extending from -300 to $+300 \mathrm{~km} \mathrm{~s}^{-1}$ for each of the lines. Note that the C IV doublet is in emission as observed in other similar stars.

We search for periodicity in the equivalent widths of the UV data of the CIV, Si IV, N V and Al III lines in intervals

\footnotetext{
1 MIDAS is distributed by the European Southern Observatory.

${ }^{2}$ IRAF is distributed by the National Optical Astronomy Observatories, which is operated by the Association of Universities for Research in Astronomy (AURA), Inc., under cooperative agreement with the National Science Foundation.
} 

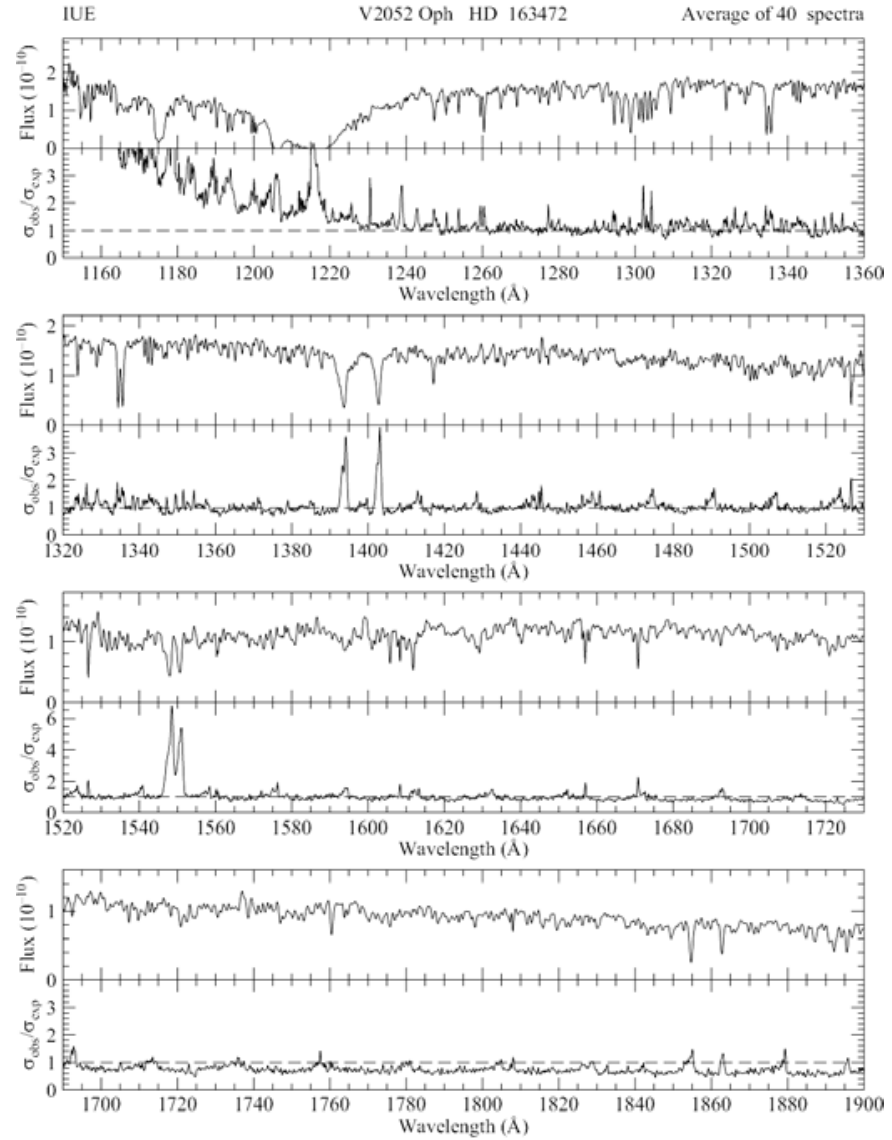

Fig. 1. Mean spectrum of 40 IUE spectra (top panels), together with the detected variability (lower panels). The repetitive pattern in the variability signature is due to imperfect correction of the échelle-order overlap regions.

with variability signature exceeding unity. The final result is obtained with a least-square method which uses weights equal to the inverse of the error bars assigned to each datapoint. With user-supplied initial starting values for the free parameters a steepest descent technique then searches for the lowest mimimum of the $\chi^{2}$. The variance matrix provides the formal errors in the parameters. A single sinusoid does not fit the data. Therefore we use the following function:

$f(t)=a+b \sin \left(2 \pi\left(\frac{t}{P}+\phi_{1}\right)\right)+c \sin \left(2 \pi\left(\frac{t}{P / 2}+\phi_{2}\right)\right)$.

The parameters of the best solution for the C IV doublet, with a reduced $\chi^{2}=0.45$, are: $a=1.09 \pm 0.04 \AA, b=1.64 \pm 0.07 \AA$, $\phi_{1}=0.54 \pm 0.03, c=0.81 \pm 0.07 \AA, \phi_{2}=0.38 \pm 0.05$ and a period $P=3.638833 \pm 0.000003 \mathrm{~d}$, where we used values of $t$ relative to the first observation. The very high precision in the period is due to the three early observations obtained in 1981-82, which extended the coverage over more than 1400 cycles, and which allowed us to select the best out of two periods with nearly equal $\chi^{2}$ by considering the phase dependence of the individual profiles. All four doublet profiles of C IV, Si IV, N V and Al III are modulated with this same period, which is identified with the rotation period of the star. With this analytic description the epoch at minimum in $E W$
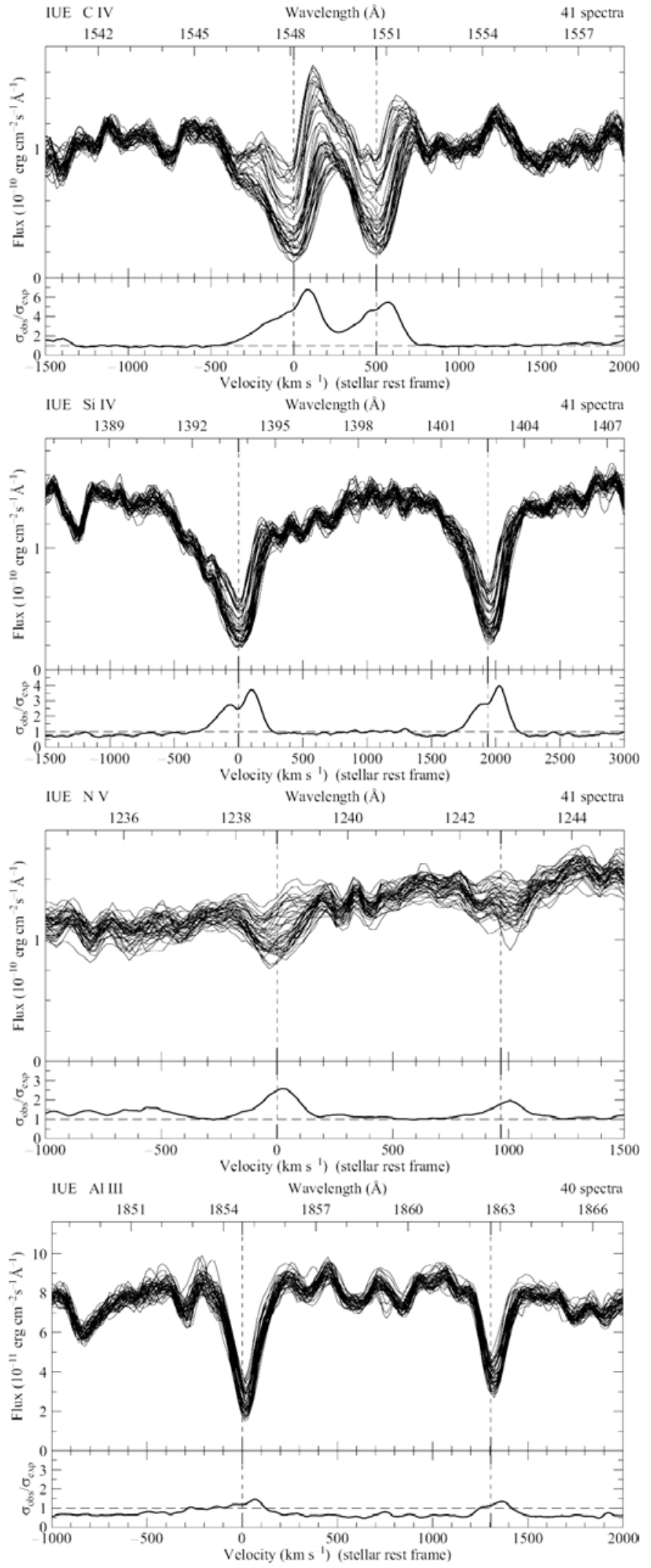

Fig. 2. Variations of the line profiles in the UV data of the CIV, Si IV, $\mathrm{NV}$ and $\mathrm{Al}$ III lines. Fluxes are normalized to the average profile.

could be derived, which we define as the zero phase of the rotation. We find $\operatorname{HJD}\left(E W_{\min }\right)=2447383.89 \pm 0.07$. 


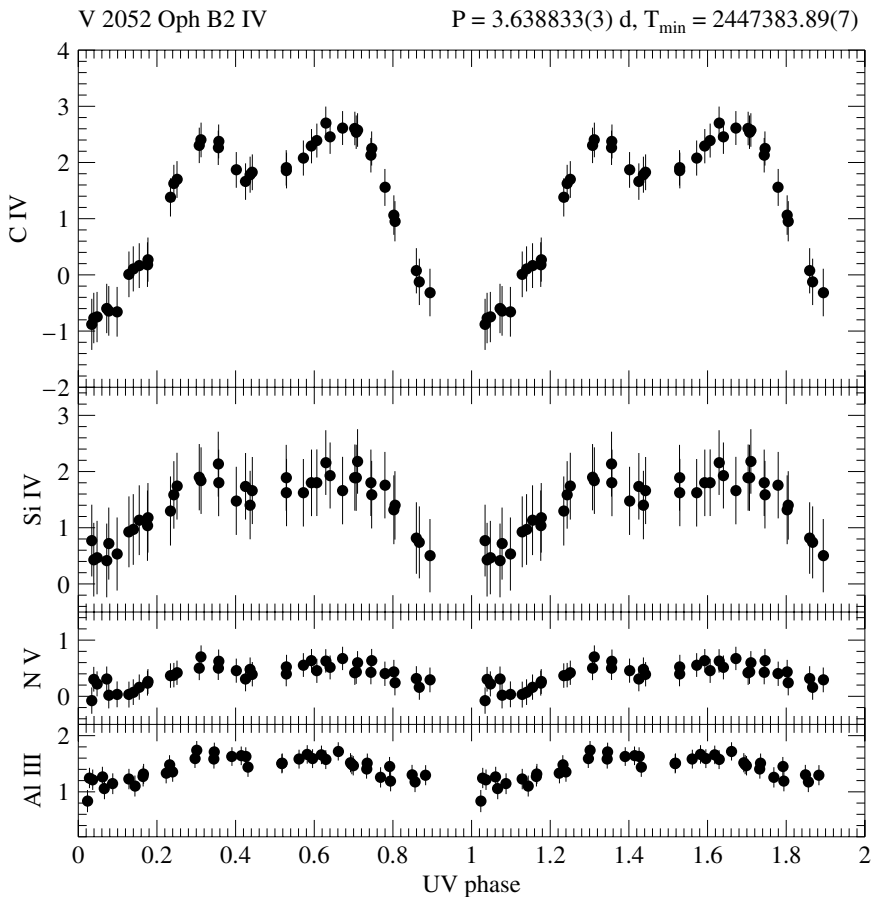

Fig. 3. Variations of the equivalent width (in $\AA$ ) from the UV data of the SiIV, CIV, NV and Al III lines, folded in phase with the rotational period. The $E W$ were measured in the following intervals: C IV in $[-450,750] \mathrm{km} \mathrm{s}^{-1}$ at $10^{-10} \mathrm{erg} \mathrm{cm}^{-2} \mathrm{~s}^{-1} \AA^{-1}$, SiIV in $[-400,2200] \mathrm{km} \mathrm{s}^{-1}$ at $10^{-10} \mathrm{erg} \mathrm{cm}^{-2} \mathrm{~s}^{-1} \AA^{-1}$, N v in $[-250,200] \mathrm{km} \mathrm{s}^{-1}$ at $1.3 \times 10^{-10} \mathrm{erg} \mathrm{cm}^{-2} \mathrm{~s}^{-1} \AA^{-1}$ and $[750,1200] \mathrm{km} \mathrm{s}^{-1}$ at $1.5 \times 10^{-10} \mathrm{erg} \mathrm{cm}^{-2} \mathrm{~s}^{-1} \AA^{-1}$, Al III in $[-200,200] \mathrm{km} \mathrm{s}^{-1}$ at $9 \times 10^{-11} \mathrm{erg} \mathrm{cm}^{-2} \mathrm{~s}^{-1} \AA^{-1}$ and [1150, $1550] \mathrm{km} \mathrm{s}^{-1}$ at $8.5 \times 10^{-11} \mathrm{erg} \mathrm{cm}^{-2} \mathrm{~s}^{-1} \AA^{-1}$.

The equivalent width measurements (in $\AA$ ) of the C IV, Si IV, N V and Al III lines folded in phase with the rotational period are shown in Fig. 3. Like in $\beta$ Cep, the $E W$ of these lines folded in phase shows two unequal minima, the deeper corresponding to maximum emission, and two equal maxima, corresponding to maximum absorption.

\section{V 2052 Oph}

\section{1. v sin i determination}

The $v \sin i$ value usually quoted for V 2052 Oph is $120 \mathrm{~km} \mathrm{~s}^{-1}$ (Jerzykiewicz 1972) but it has been estimated with poor accuracy from low-resolution spectra in 1972. Wolff et al. (1982) found $v \sin i=60 \mathrm{~km} \mathrm{~s}^{-1}$ from visual comparison with a rotational standard. More recently Smith \& Groote (2001) derive $100 \mathrm{~km} \mathrm{~s}^{-1}$ from a comparison of models.

We determine the value of $v \sin i$ by applying a Fourier transform analysis (Gray 1976) to the mean line profile of the HeI 4713, 4921, 5016 and 5876 and Si III 4553 and 4568 lines obtained at TBL. A limb darkening coefficient $\epsilon=$ 0.4 is adopted. The results are reported in Table 3 . We obtain $v \sin i=63 \pm 2 \mathrm{~km} \mathrm{~s}^{-1}$.
Table 3. $v \sin i$ determination using a Fourier transform analysis.

\begin{tabular}{lllllll}
\hline \hline & He I & & & \multicolumn{3}{c}{ Si III } \\
\hline Line & 4713 & 4921 & 5016 & 5876 & 4553 & 4568 \\
$v \sin i\left(\mathrm{~km} \mathrm{~s}^{-1}\right)$ & 62.7 & 64.4 & 62.9 & 64.0 & 63.3 & 62.6 \\
\hline
\end{tabular}

\subsection{Stellar parameters}

The star V 2052Oph is a $V=5.85$ star with a spectral type B2 IV-V (Rountree Lesh 1968). UV classification gives B2 IV (Rountree \& Sonneborn 1991) by comparison with the star $\zeta$ Cas.

Two previous studies indicate rather different fundamental parameters. An equivalent width study performed by Wolff \& Heasley (1985) led to an effective temperature of about $T_{\text {eff }}=23000 \mathrm{~K}$, for a gravity of $\log g=4.2$ and a $\mathrm{He} / \mathrm{H}$ ratio of 0.085 . However, Smith \& Groote (2001) found that the flux of the UV IUE spectra implies a higher effective temperature $T_{\text {eff }}=26000 \mathrm{~K}$ for a gravity fixed at $\log g=4.0$.

In our first attempt we determined the stellar effective temperature and superficial gravity assuming a solar $\mathrm{He} / \mathrm{H}$ ratio (0.1) by fitting several He I lines (4026, 4387, $4921 \AA$ ), observed with the VLT. We built up line profile grids using NLTE model atmospheres computed with TLUSTY (Hubeny \& Lanz 1995, and references therein) for different values of effective temperature and gravity. In these calculations $\mathrm{H}, \mathrm{He}, \mathrm{C}$, $\mathrm{N}, \mathrm{O}$ and $\mathrm{Si}$ were treated in NLTE. Except for O I which was treated with the MODION IDL package, the atomic models we used are those proposed by Hubeny \& Lanz on TLUSTY's web site $^{3}$.

For the solar $\mathrm{He} / \mathrm{H}$ case, we are able to model simultaneously all the He I line profiles with $T_{\text {eff }}=21991 \mathrm{~K}, \log g=$ 3.98 and $v \sin i=58 \mathrm{~km} \mathrm{~s}^{-1}$. This is very close to the results obtained by Wolff \& Heasley (1985). In this configuration, nitrogen and oxygen have solar abundances (Grevesse \& Sauval 1998) while carbon and silicon are depleted. Although the computed line wings are not as extended as the observations, a good agreement is obtained for the $\mathrm{H} \alpha$ line, but the Si II 4128 and 4132 (VLT) and Si III 4553, 4568 and 4575 (TBL) lines could not be fitted using the same silicon abundances. We find that, in order to fit all the silicon and neutral helium lines simultaneously with the same abundance, we need to increase the effective temperature and, consequently, increase the $\mathrm{He} / \mathrm{H}$ ratio.

To estimate the helium abundance, we extend our flux grids using computations made by Zboril (2000) with TLUSTY for different values of the $\mathrm{He} / \mathrm{H}$ ratio and available at the CDS. The most consistent model, according to the helium and silicon line profiles, is obtained for $T_{\text {eff }}=25200 \mathrm{~K}, \log g=4.2$, $v \sin i=60 \mathrm{~km} \mathrm{~s}^{-1}$ and $\mathrm{He} / \mathrm{H}=0.21$. These values are much closer to the ones proposed by Smith \& Groote (2001). They allow us to fit the Si II and Si III lines simultaneously. Moreover they also allow to fit the continuum slope of the short wavelength IUE spectra adopting the mean reddening law derived by Cardelli et al. (1989) and $E(B-V)=0.30$, derived from Papaj et al. (1990) and consistent with the

\footnotetext{
${ }^{3}$ http://tlusty.gsfc.nasa.gov
} 

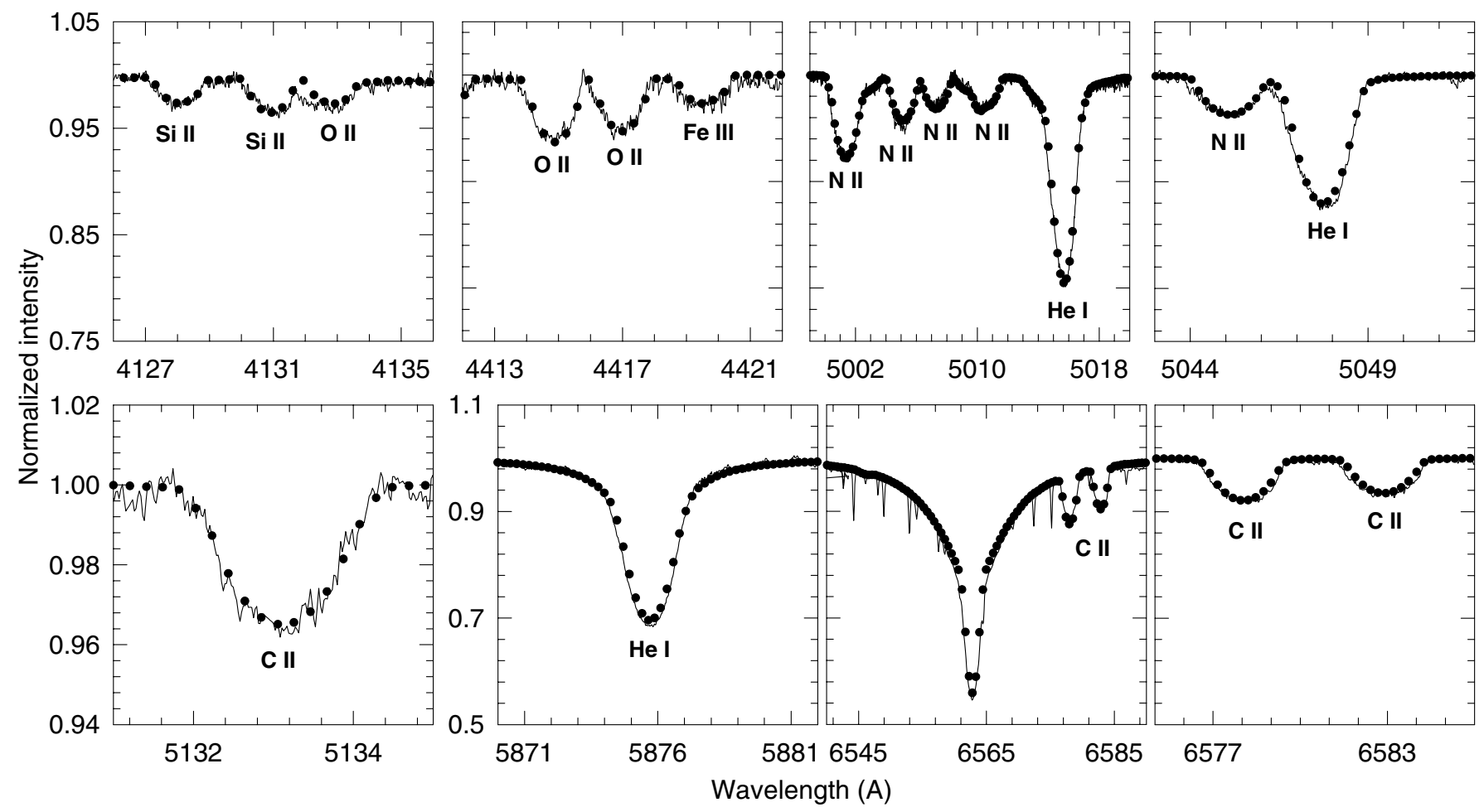

Fig. 4. Model fit result for the lines used to determine the stellar parameters. The dots representing the synthetic spectra are overplotted on the observations (solid lines).

Table 4. Stellar parameters of the star V 2052 Oph (HD 163472, HR 6684).

\begin{tabular}{llll}
\hline \hline & Model & Literature & Ref. \\
\hline Spectral type & B1 V & B2 IV-V & RL68 \\
$V$ & & $5.83-5.87$ & Hip \\
Distance (pc) & $290 \pm 50$ & $254_{-50}^{+83}$ & Hip \\
$T_{\text {eff }}$ & $25200 \pm 1100$ & 26000 & SG01 \\
$\log g$ & $4.2 \pm 0.11$ & 4.2 & W85 \\
$M / M_{\odot}$ & $10.1 \pm 0.6$ & & \\
$R / R_{\odot}$ & $4.1 \pm 0.2$ & 6.0 & BB77 \\
$\log L / L_{\odot}$ & $3.81 \pm 0.10$ & & \\
$v \sin i\left(\mathrm{~km} \mathrm{~s}^{-1}\right)$ & $60 \pm 4$ & 63 & Sect. 4.1 \\
$P_{\text {rot }}(\mathrm{d})$ & & 3.638833 & Sect. 3 \\
& & \pm 0.000003 & \\
$i\left({ }^{\circ}\right)$ & $71 \pm 10$ & & \\
\hline
\end{tabular}

RL68: Rountree Lesh (1968), Hip: Perryman et al. (1997), W85: Wolff \& Heasley (1985), SG01: Smith \& Groote (2201), BB77: Beeckmans \& Burger (1977).

determination $E(B-V)=0.33$ from Diplas \& Savage (1994). As far as the red $\mathrm{He} \mathrm{I}$ and $\mathrm{H} \alpha$ lines are concerned, the agreement between observations and theory are better than with the solar value (Fig. 4). The value of $v \sin i$ is compatible with the one derived in the previous Sect. 4.1. With this effective temperature, V 2052 Oph would be a B1 V star rather than a B2 IV-V star. The mismatch with the MK classification is probably due to the unusual strength of the He I lines.
Using the evolutionary tracks of Schaller et al. (1992), we derive $M=10.1 M_{\odot}$ for the mass and $R=4.1 R_{\odot}$ for the radius. For a star with such a mass, the critical break-up rotational velocity is about $580 \mathrm{~km} \mathrm{~s}^{-1}$. The critical radius $R_{\text {crit }}=5.6 R_{\odot}$ is obtained using Roche's model. The angular velocity $\omega$ is about $15 \%$ of the angular critical velocity. The effects of gravity darkening are therefore negligible. Using $T_{\text {eff }}$ and $R$, we also obtain the luminosity of the $\operatorname{star} \log L / L_{\odot}=3.81$.

Using the above parameters, $E(B-V)=0.30$ (Papaj et al. 1990) and comparing the flux of the IUE spectra to the flux of the models, we obtain a distance $d=290 \pm 50 \mathrm{pc}$, which is compatible with the distance derived from the Hipparcos parallax of $254_{-50}^{+83}$ pc. In Sect. 3 we showed that the rotational period is $P_{\text {rot }}=3.64 \mathrm{~d}$. With $v \sin i=60 \mathrm{~km} \mathrm{~s}^{-1}$, we obtain $i=71^{\circ}$ and $v=63 \mathrm{~km} \mathrm{~s}^{-1}$. The stellar parameters are summarized in Table 4.

\subsection{Chemical composition}

With the model presented above we can also determine the chemical composition of the star by fitting individual lines with Tlusty (Hubeny \& Lanz 1995, and references therein). The H, $\mathrm{He}, \mathrm{C}, \mathrm{N}$ and $\mathrm{O}$ ions are treated in NLTE, while the other ions are treated in LTE. Again, the atomic models are those proposed on Tlusty's web site, except for O I.

To determine the abundances of carbon, nitrogen, oxygen and silicon, we select from the NIST compilation database the transitions with the most accurate oscillator strengths (10\% or better). We update Kurucz line lists with these new values and fit the selected observed spectral range (Fig. 4) using the least 
Table 5. Chemical composition of V 2052 Oph. Column 2 gives the number $N$ of lines for each ion, used to determine the chemical abundance.

\begin{tabular}{llr}
\hline \hline Ion & $N$ & \multicolumn{1}{c}{$\log \left(\epsilon_{N} / \epsilon_{\odot}\right)$} \\
\hline $\mathrm{He}_{\mathrm{I}}$ & 4 & $0.32 \pm 0.05$ \\
$\mathrm{C}_{\text {II }}$ & 3 & $-0.13 \pm 0.04$ \\
$\mathrm{~N}_{\text {II }}$ & 6 & $0.10 \pm 0.06$ \\
$\mathrm{O}_{\text {II }}$ & 4 & $-0.31 \pm 0.11$ \\
$\mathrm{Si}$ II + III & 5 & $0.01 \pm 0.10$ \\
\hline
\end{tabular}

squares method. We adopt a $2 \mathrm{~km} \mathrm{~s}^{-1}$ microturbulence velocity, as proposed in Andrievsky et al. (1999), which allows to fit simultaneously the different studied transitions. The results of our fits are given in Table 5 where $\epsilon_{N} / \epsilon_{\odot}$ denotes the abundance in the star relative to the abundance in the Sun as compiled by Grevesse \& Sauval (1998).

We found that V 2052 Oph is He-strong and O-weak. The relative $\mathrm{C}, \mathrm{N}$ and $\mathrm{O}$ abundances we derive follow the same pattern as the one described by Zboril \& North (2000) in their LTE spectral analysis for a sample of $21 \mathrm{He}$-strong stars. The carbon abundance is twice lower than their mean value for this kind of objects, probably due to strong departures from LTE for the C II 4267 line they used, as noted by the authors.

\section{Pulsations}

\subsection{Periodicity search methods}

We performed two methods to search for periodicity in the data used in this paper: the Restricted Local Cleanest (RLC, based on Foster 1995, 1996, developed by Emilio 1997 and Domiciano de Souza Jr. 1999 and applied in Domiciano de Souza et al. 2000) and a Least-Squares (LS) fitting methods.

In each wavelength bin, the RLC method searches for 20 frequencies in a predefined range and computes all possible models with 4 frequencies. Comparing the power of each of these models, it selects 7 optimal frequencies while suppressing the aliases. A Local Cleanest (Foster 1995) is then applied to these 7 values to finetune the final frequencies (for more details see Neiner et al. 2002).

With the LS method, we look at the whole line at the same time, i.e. all wavelength bins at once, to determine which frequencies describe the variations in the best way. After a first frequency is found, the data are prewhitened, and the program looks for the next frequency in the residual spectra. This procedure is repeated several times.

\subsection{Line profiles}

The 111 individual spectra obtained at TBL in 2000 and the 407 ones obtained in 2001 are used to search for periodicity in the line profile variations of the He I 4713, 4921, 5016 and 5876 and Si III 4553 line. We use the two methods described above. The search is separately performed on both
Table 6. Frequencies detected from the analysis of different lines using the RLC method for the 2001 data. The frequencies obtained with the LS method are shown in boldface.

\begin{tabular}{lllll}
\hline \hline Line & \multicolumn{4}{l}{ Frequencies $\left(\mathrm{c} \mathrm{d}^{-1}\right)$} \\
& $f_{1}$ & $f_{2}$ & $f_{3} \sim 2 f_{\text {rot }}$ & $f_{4} \sim f_{\text {rot }}$ \\
\hline He I 4713 & $7.15 / 7.15$ & $6.86 / 6.83$ & $0.50 / \mathbf{0 . 5 5}$ & $0.27 / \mathbf{0 . 2 9}$ \\
He I 4921 & $7.14 / 7.15$ & $\mathbf{6 . 8 3}$ & $0.50 / \mathbf{0 . 5 5}$ & $0.28 / \mathbf{0 . 2 8}$ \\
He I 5016 & $7.14 / 7.15$ & $6.82 / \mathbf{6 . 8 3}$ & $0.51 / \mathbf{0 . 5 5}$ & $0.27 / \mathbf{0 . 2 9}$ \\
He I 5876 & $7.14 / 7.15$ & 6.84 & $0.50 / \mathbf{0 . 5 5}$ & $0.27 / \mathbf{0 . 2 2}$ \\
Si III 4553 & $7.14 / 7.15$ & 6.81 & $0.50 / \mathbf{0 . 5 5}$ & $0.25 / \mathbf{0 . 2 9}$ \\
\hline
\end{tabular}

sets of data and on the combined dataset $2000+2001$. As the data taken in 2000 are of lower quality and badly sampled in time because of bad weather conditions, including them does not improve the results obtained with the data taken in 2001 only. Therefore we will present here the results obtained with the 2001 data only. Due to the timebase of the observations, frequencies lower than $0.05 \mathrm{c} \mathrm{d}^{-1}$ cannot be detected. For the same reason, two frequencies closer than $0.025 \mathrm{c} \mathrm{d}^{-1}$ cannot be distinguished.

For all the studied lines, three frequencies are detected: (i) a very strong frequency stands out at $f_{1}=7.145 \pm$ $0.005 \mathrm{c} \mathrm{d}^{-1}$, corresponding to the well-known pulsation period $P_{1}=3 \mathrm{~h} 21 \mathrm{~min}$;

(ii) a second frequency is detected at $f_{2}=6.82 \pm 0.02 \mathrm{c} \mathrm{d}^{-1}$ corresponding to a period $P_{2}=3 \mathrm{~h} 31 \mathrm{~min}$;

(iii) a third frequency is present at $f_{3}=0.55 \pm 0.02 \mathrm{c} \mathrm{d}^{-1}$. This corresponds to twice the rotational frequency $f_{\text {rot }}=0.27 \mathrm{c} \mathrm{d}^{-1}$. This is the first detection of multiperiodicity in V 2052 Oph. The detected frequencies for each line with the two methods of analysis are summarized in Table 6.

Periodograms, together with the mean line profile, are shown in Fig. 5. Other frequencies also appear in the periodograms and are due either to the window spectrum or to the combination of the real frequencies $\left(f_{1}, f_{2}, f_{3}\right.$ and $\left.f_{\text {rot }}\right)$ with each other and with the window spectrum.

The power spectrum obtained for the He I 5016 line profile, showing the three frequencies and $f_{\text {rot }}$, is plotted in Fig. 6 . In the top panel, the power spectrum of the He I 4921 line profile, which is slightly different in this frequency region, is overplotted.

\subsection{1. $f_{1}=7.15 \mathrm{c} \mathrm{d}^{-1}$ : Radial mode}

A very strong frequency is detected at $f_{1}=7.145 \mathrm{c} \mathrm{d}^{-1}$. This frequency was already known in the literature (e.g. Jerzykiewicz 1972) and was also detected by the Hipparcos satellite. Cugier et al. (1994) proposed that this belongs to a radial pulsation mode.

Looking at the phase and power of this frequency along the line profile (Fig. 7), we can confirm that $f_{1}$ is due to a pulsation mode with $l=0$, as the phase shows no significant slope (see Schrijvers et al. 1997). The phase is also coherent outside the 


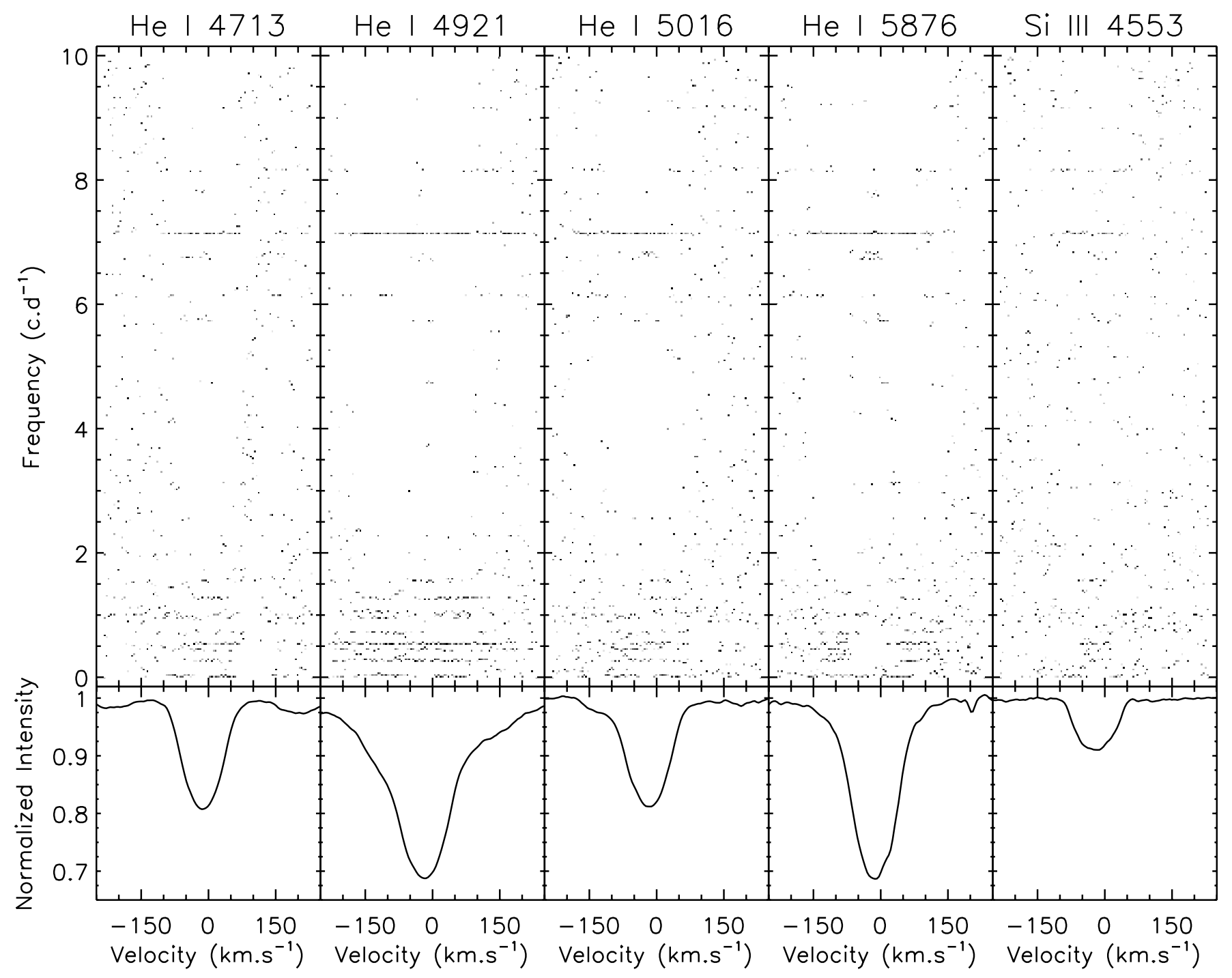

Fig. 5. For each studied line, the upper panel shows the periodogram obtained with the RLC method, together with the mean line profile in the lower panel.

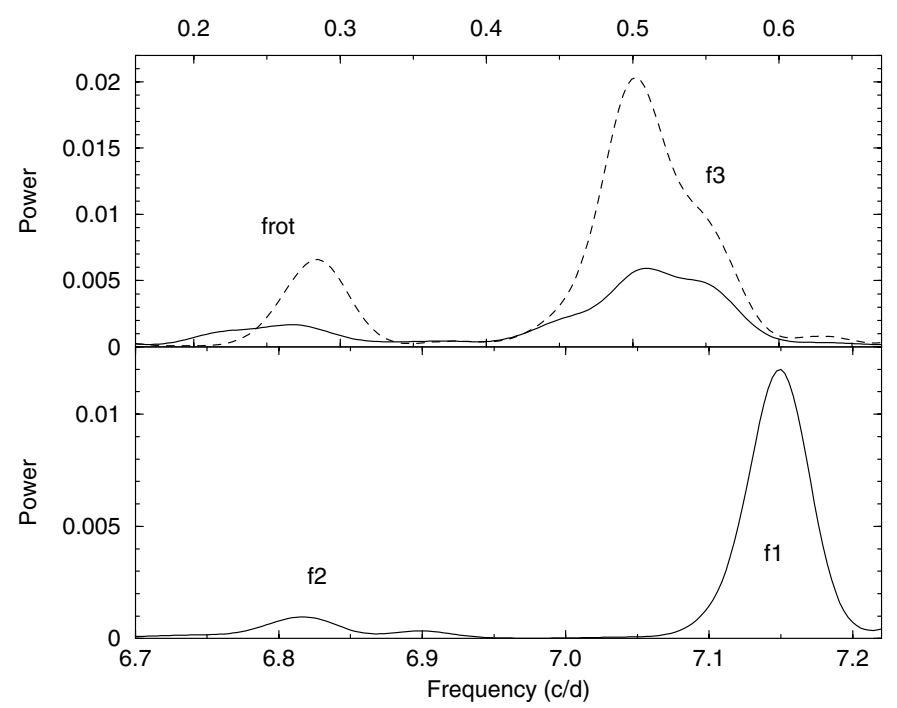

Fig. 6. Example of a power spectrum obtained for the He I 5016 line profile. In the top panel the power spectrum of the He I 4921 line profile is overplotted with a dashed line. line in some cases, due to the presence of other weak lines (e.g. blue side of the He I 4921 line) which also pulsate with $f_{1}$.

A greyscale plot of the spectra for several lines, from which the mean line profile has been subtracted, is presented in Fig. 8 in phase with the frequency $f_{1}$.

\subsection{2. $f_{2}=6.82 \mathrm{c} \mathrm{d}^{-1}$ : Non-radial mode}

Although it is about 20 times less powerful than $f_{1}$, a frequency is detected at $f_{2}=6.82 \mathrm{c} \mathrm{d}^{-1}$. Looking at the slope of the phase and power of this frequency (Fig. 9), we can derive the pulsation degree $l$ (see Schrijvers et al. 1997). We obtain that $f_{2}$ corresponds to a non-radial pulsation mode with $l=3$ or 4 .

A greyscale plot of the He I 4713, 4921, 5016 and 5876 and Si III 4553 lines, obtained after prewhitening the frequency $f_{1}$, is presented in Fig. 10 in phase with the frequency $f_{2}$. The prewhitening was done by computing the phase and power of the frequency $f_{1}$ with a least squares fit for all wavelength bins simultaneously (see LS description in Sect. 5.1) at each time. 

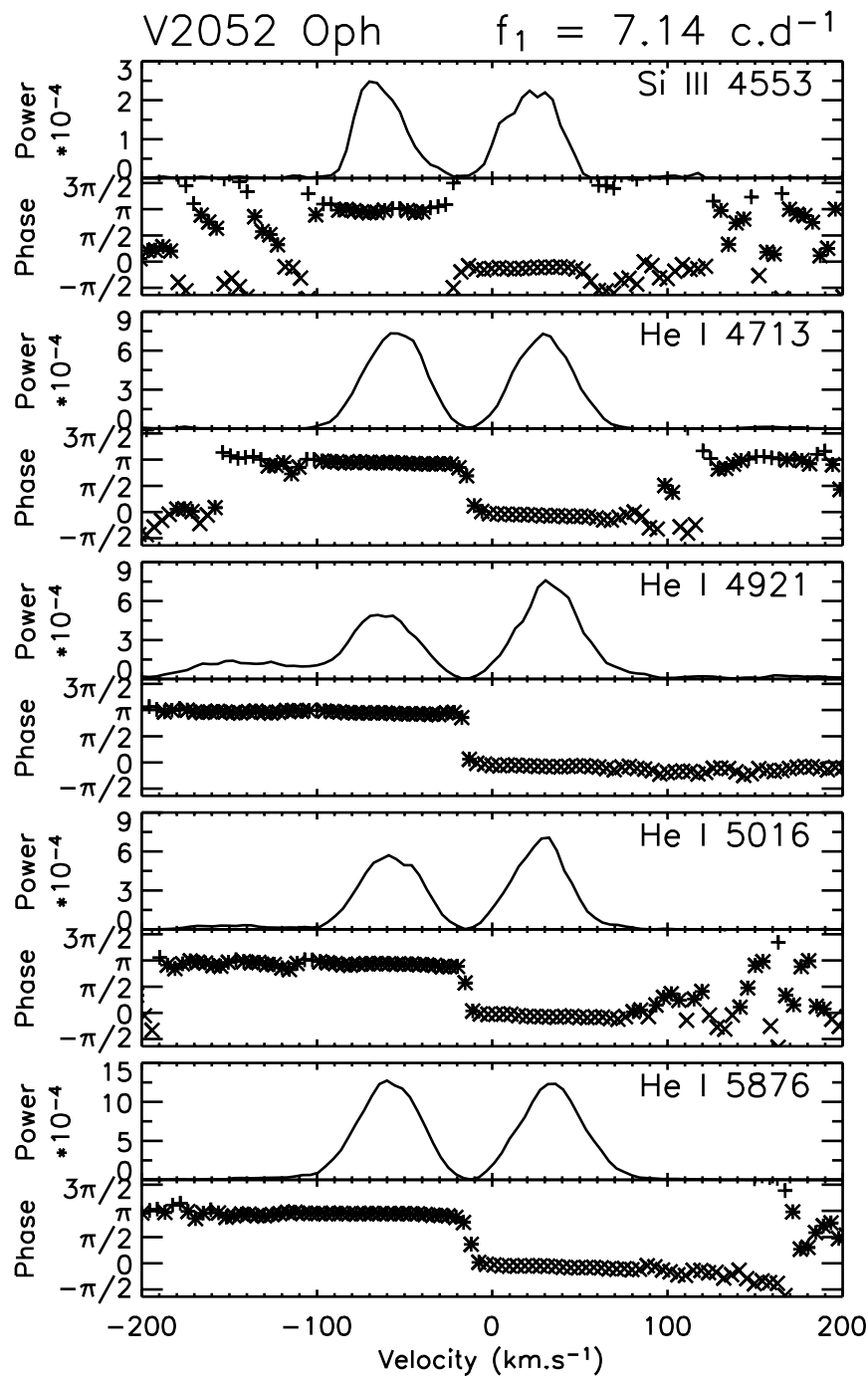

Fig. 7. Power and phase of the frequency $f_{1}=7.145 \mathrm{c} \mathrm{d}^{-1}$ along the line profiles of the Si III 4553 and the He I 4713, 4921, 5016 and 5876 lines.

The spectra, minus the mean line profile, were then corrected from this two-dimensional sine curve, in wavelength and time.

\subsection{3. $f_{3}=0.55 \mathrm{c} \mathrm{d}^{-1}$ : Rotation}

A frequency is detected at $f_{3}=0.55 \mathrm{c} \mathrm{d}^{-1}$. This frequency cannot be due to a window effect, as it does not appear in the window power spectrum. Interestingly, the frequency $f_{3}$ corresponds to twice the rotational frequency $f_{\text {rot }}=0.27 \mathrm{c} \mathrm{d}^{-1}$ derived from the UV analysis. The rotational frequency itself, $f_{\text {rot }}$, is also detected but with a weaker power. See Sect. 7 for further discussion.

\subsection{Radial velocity}

Radial velocities are measured using a centroid set at the intensity 0.8 . We study the radial velocity variations of the He I 4921 and 5876 lines. They are dominated by the frequency $f_{1}$ as shown in Fig. 11 for the He I 4921 line. In this figure we



Fig. 8. Greyscale plot of the spectra taken in 2001, folded in phase with the frequency $f_{1}=7.145 \mathrm{c} \mathrm{d}^{-1}$.

overplotted parts of the best fit sine wave imposing the frequency $f_{1}$. The same behavior is observed for the He I 5876 line. The semi-amplitude of the fit is about $9 \mathrm{~km} \mathrm{~s}^{-1}$ for He I 4921 and 5876 (see also Fig. 13).

Folding the data in phase with $f_{1}$ (Fig. 12) shows that the scatter of points $\left( \pm 2 \mathrm{~km} \mathrm{~s}^{-1}\right)$ is higher than the precision of the measurements compared to the individual best-fit sine curves (Fig. 11) and implies the presence of at least one other frequency.

We measure the mean value and amplitude of the variations for each night of observations and investigate the variations of these measurements. Although it is difficult to extract a frequency with any method we tried, due to the small amount of points and small amplitude of variations, folding the data in phase with $f_{3}=0.55 \mathrm{c} \mathrm{d}^{-1}$ shows that this frequency is present in the radial velocity measurements (e.g. Fig. 13 for the He I 4921 line). 

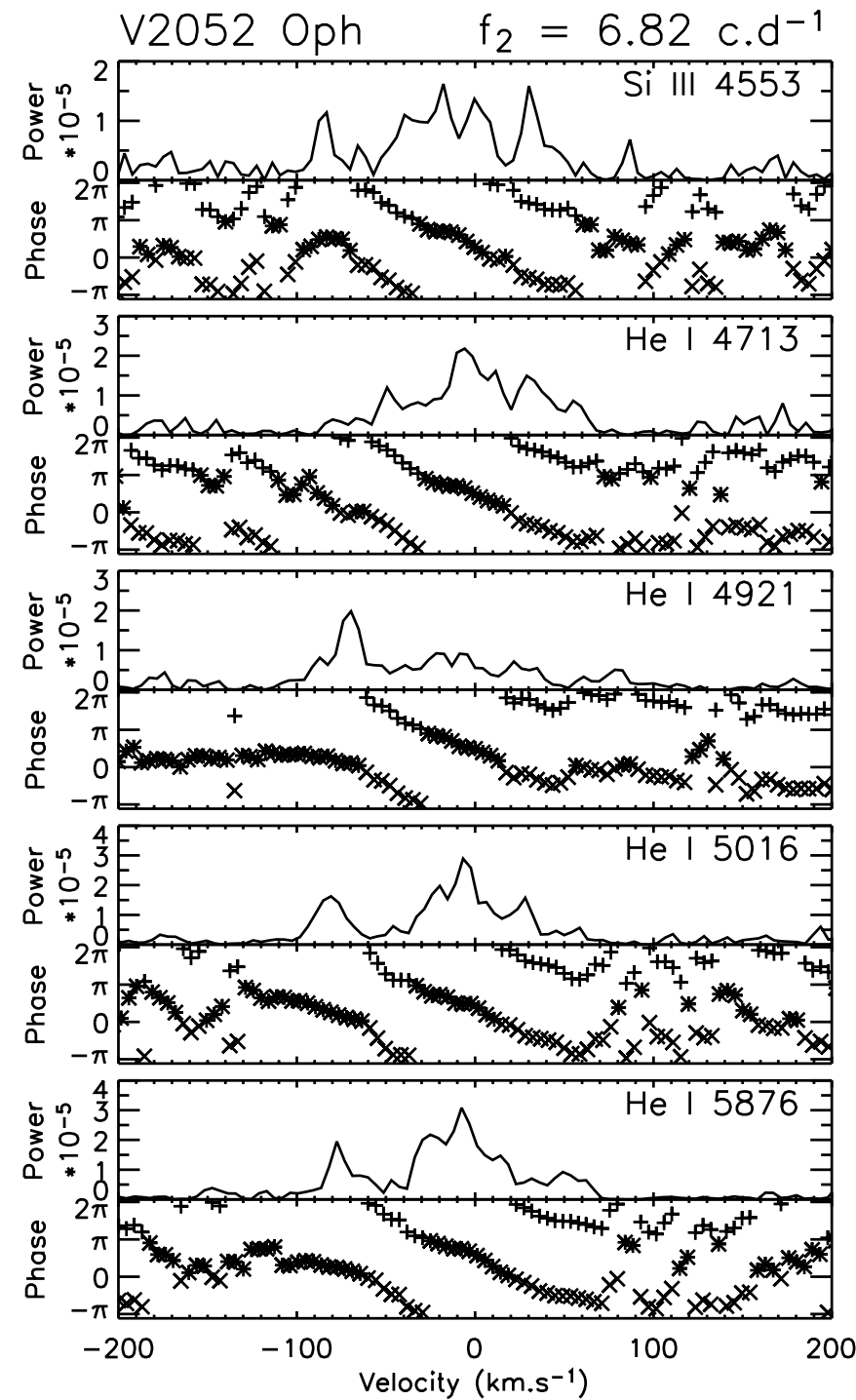

Fig. 9. Power and phase of the frequency $f_{2}=6.82 \mathrm{c} \mathrm{d}^{-1}$ along the line profiles of the Si III 4553 and the He I 4713, 4921, 5016 and 5876 lines.

\subsection{Minimum intensity}

We study the minimum intensity variations of the He I 4921 and 5876 lines. The minimum intensity $I_{\min }$, normalized to the continuum intensity $I_{\mathrm{c}}=1$, is obtained for each spectrum by taking the point $P$ of minimum intensity and fitting a parabola to the 21 points around this minimum ( $P \pm 10$ points). Using a LS method to search for periodicity, we detect the frequencies $f_{\text {rot }}, f_{1}, f_{2}$ and $f_{3}$ for almost all the studied lines.

Folding the minimum intensity measurements of the He I lines in phase with the rotational frequency (Fig. 14) reveals two minima and two maxima, similar to the equivalent width measurements of the UV stellar wind lines. Interestingly, the Si III 4553 line varies in the opposite way compared to the He I lines. Note that the He I 5876 line also seems to show a different behavior, probably due to saturation.



Fig. 10. Greyscale plot of the spectra taken in 2001, prewhitened from the frequency $f_{1}$, folded in phase with the frequency $f_{2}=6.82 \mathrm{c} \mathrm{d}^{-1}$.

\section{Magnetic field}

\subsection{Direct measurements}

Due to the relative faintness of the star the magnetic measurements have relatively large error bars. Table 7 (only available in electronic form) shows the longitudinal magnetic field value $B$, its error bar $\sigma B$, the null polarisation $N$ and its error $\sigma N$. The null polarisation $N$ gives an indication of the pollution by non-stellar effects and should be zero for a perfect measurement. It is produced by associating the four subexposures of one magnetic measurement in the same way as for creating the Stokes V profile, except that the two last subexposures are exchanged. In the following, only measurements for which $N<\sigma N$ are used. Moreover, for the data taken in 2000, bad weather conditions and fringes also decrease the quality of the data, making them less reliable than the 2001 data. Exposure times were chosen between 7 and 12 min, i.e. much shorter 


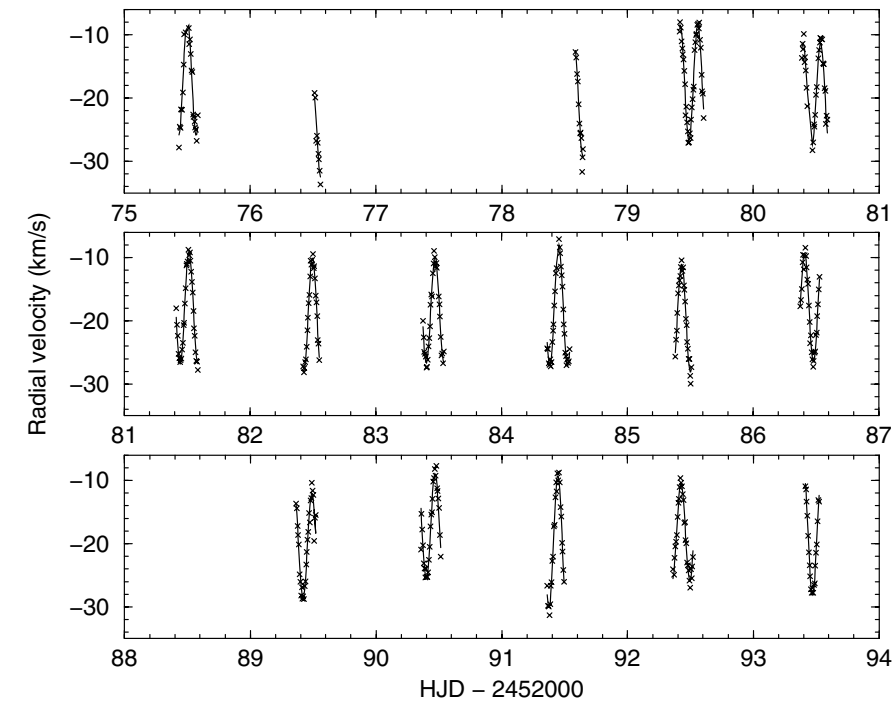

Fig. 11. Radial velocity measurements of the He I 4921 line. Best fit sine waves with the frequency $f_{1}$, determined for each night, are overplotted.

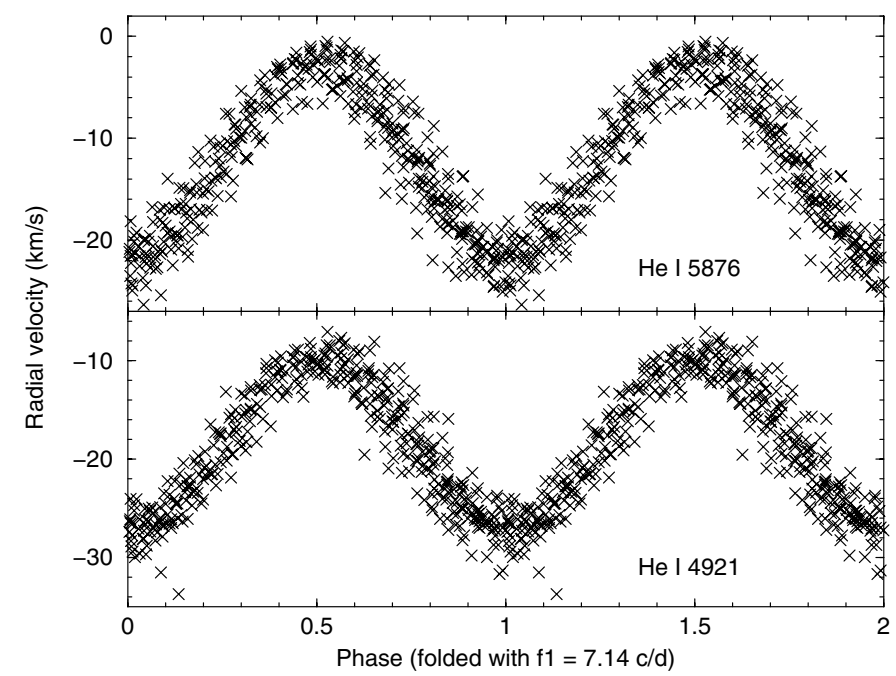

Fig. 12. Radial velocity measurements of the He I 4921 and 5876 lines, folded in phase with the frequency $f_{1}=7.145 \mathrm{c} \mathrm{d}^{-1}$.

than the pulsation period, to avoid smearing of the magnetic signal because of the pulsational effect.

Although no strong signatures appear in the individual Stokes $\mathrm{V}$ profiles, the coherent variation of the magnetic measurement in phase is an indication of the presence of a weak longitudinal field in V $2052 \mathrm{Oph}$. In the frame of the magnetic rotator model (see Shore 1987) the UV period corresponds to the rotational period of the star (see next section). The magnetic field should then vary in phase with the equivalent width of the UV resonance lines, i.e with $P \sim 3.64 \mathrm{~d}$ (see Fig. 15).

Assuming that $\mathrm{V} 2052 \mathrm{Oph}$ hosts a magnetic field and folding the magnetic data with $P_{\text {rot }}$, we calculate a best-fit sine wave through the magnetic data. This gives an amplitude of the longitudinal component of $B_{1}=92 \pm 41 \mathrm{G}$, around the average value of $B_{0}=-8 \pm 25 \mathrm{G}$ with a reduced $\chi^{2}=1.14$. This fit is shown as a dashed line in Fig. 15.

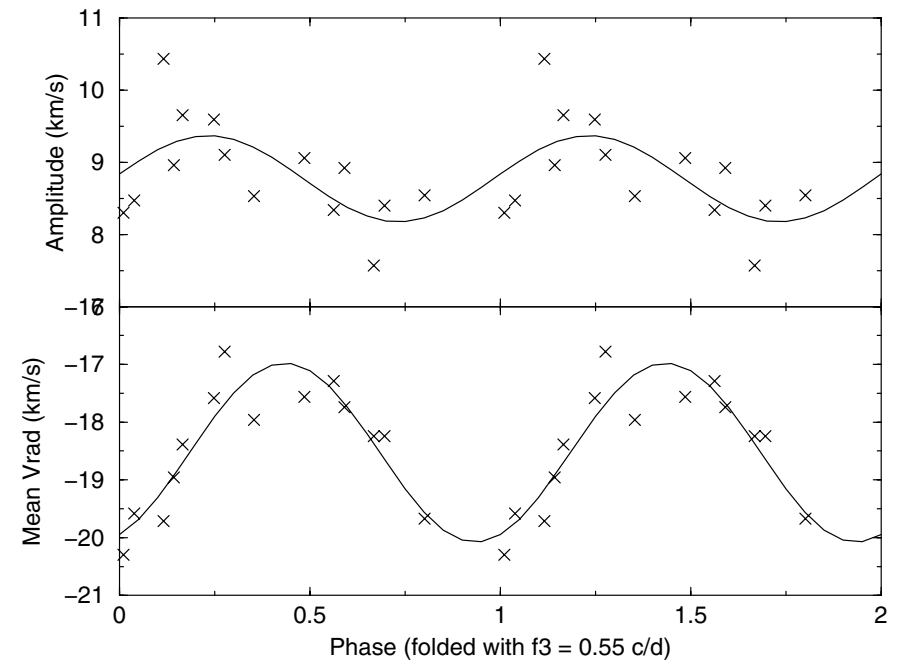

Fig. 13. Mean radial velocity and semi-amplitude for each night of the He I 4921 line, folded in phase with the frequency $f_{3}=0.55 \mathrm{c} \mathrm{d}^{-1}$.

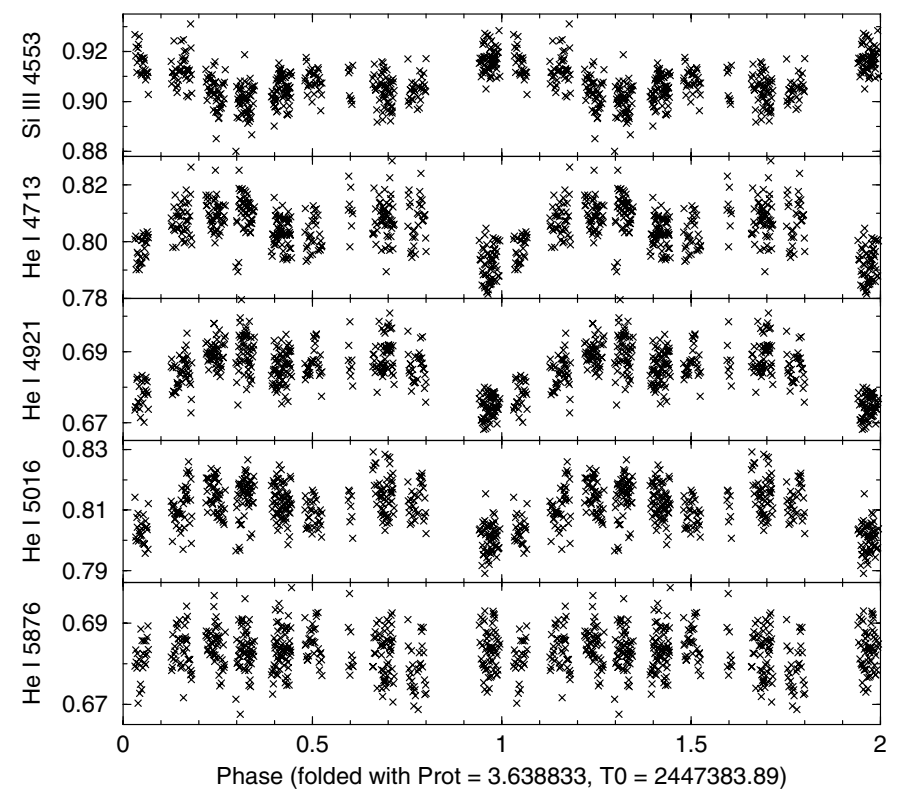

Fig. 14. Minimum intensity measurements of the Si III 4553, He I 4713, 4921, 5016 and 5876 lines, folded in phase with the rotational period $P_{\text {rot }}$.

With the derived phase we find for the ephemeris of the maximum value of the field strength: $\operatorname{HJD}(B \max )=$ $2451906.86 \pm 0.21$. Comparison with the observed $E W$ minimum of the C IV UV data, which were taken $\sim 1200$ rotational cycles prior to the magnetic data, shows that a deep $E W$ minimum is predicted at HJD $2451906.96 \pm 0.07$, which is within the uncertainties identical to the phase of maximum magnetic field. Note the striking resemblance in phase correlation with the $\beta$ Cep results (Henrichs et al. 2000; Donati et al. 2001).

\subsection{Oblique magnetic dipole}

We have found evidence for a weak varying longitudinal field in V $2052 \mathrm{Oph}$, consistent with an oblique magnetic dipole with a rotational period of about $3.64 \mathrm{~d}$. In the oblique magnetic 


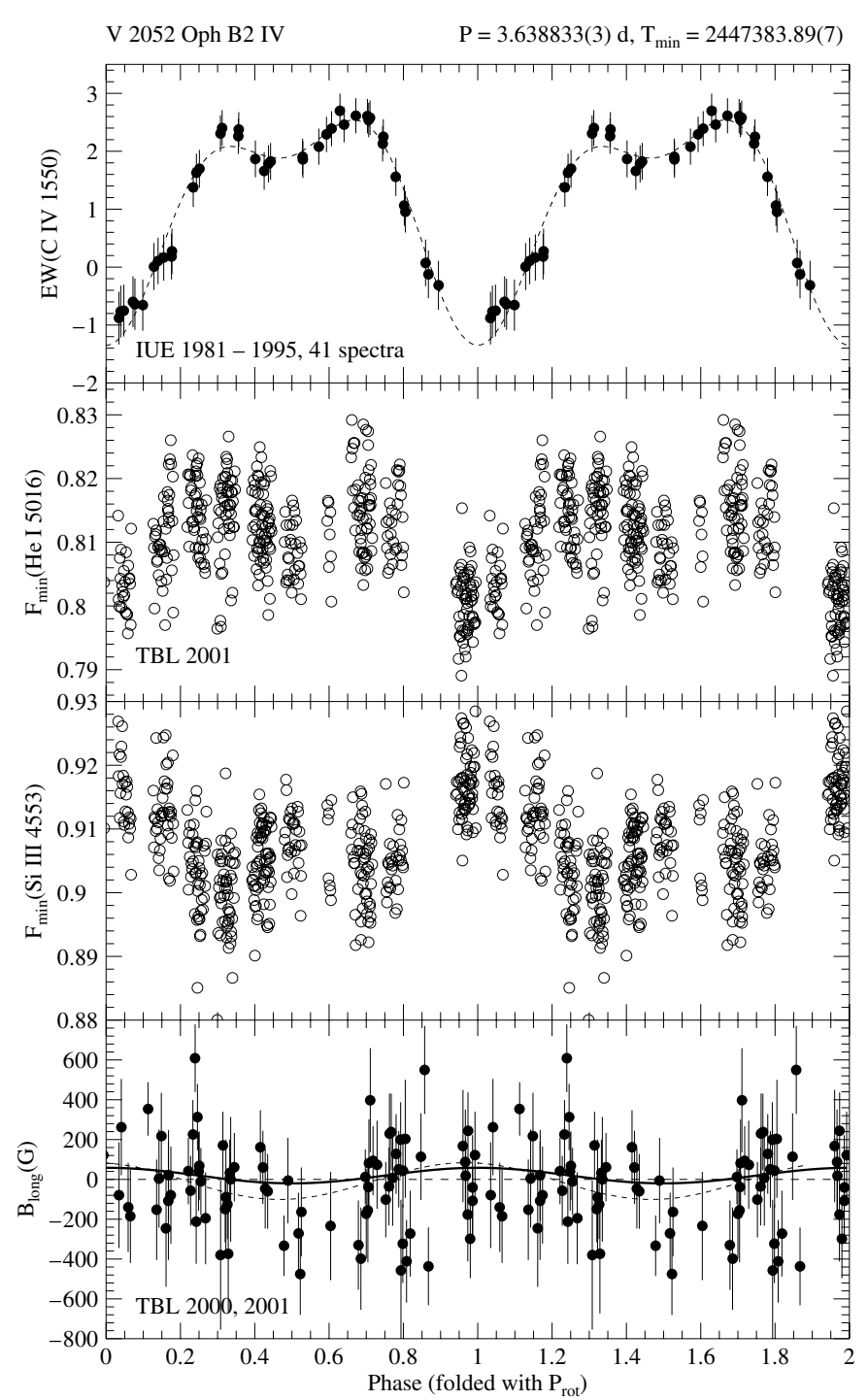

Fig. 15. Top panel: variations of the equivalent width of the UV C IV line; middle panels: variations of the minimum intensity of the He I 5016 and Si III 4553 lines; lower panel: variations of the longitudinal magnetic field; the best sine fit (dashed line) and the dipole model (solid line) are overplotted. All the plots are folded with the rotation period.

dipole model (Stibbs 1950) the axis of the dipole and the axis of rotation do not coincide. The observed stellar configuration can then be characterised by the inclination angle $i$ and by the angle $\beta$ between the magnetic axis and the rotation axis. Therefore, as the star rotates, the aspect of its visible hemisphere changes (e.g. Fig. 16). This leads to variations in various observables with the stellar rotation period, such as the shape and equivalent width of wind-sensitive UV resonance lines (see Sects. 3 and 5) and the value of the longitudinal magnetic field. See Shore (1987) for more details on the following Eqs. (2)-(4).

For a dipolar field, the ratio of the magnetic extremes $r=$ $B_{\max } / B_{\min }$ is related to the inclination angle $i$ and the angle between the magnetic and the rotation axis $\beta$ via

$r=\frac{\cos (i+\beta)}{\cos (i-\beta)}$
In the case of V $2052 \mathrm{Oph}$ we find $r=-0.83$, corresponding to $\beta=75^{\circ}$, but the large error bar in the field strength parameters actually indicates a value between $\beta=20$ and $85^{\circ}(-4.2<r<$ 1.9) for $i=71^{\circ}$ and an even larger range if the errors on $i$ are taken into account. Thus, by this method, the angle $\beta$ is not well constrained.

The observed phase difference $\Delta \phi$ between the two UV maxima is also related to the angles $i$ and $\beta$ through

$\cos \frac{\Delta \phi}{2}=\frac{1}{\tan i \tan \beta}$.

In the case of V 2052 Oph we find $\Delta \phi=0.335 \pm 0.026$. If we adopt an inclination angle $i=71 \pm 10^{\circ}$ as derived in Sect. 5, we obtain $\beta=35 \pm 18^{\circ}$, which we adopt in the following as the best value.

When two maxima are observed in the UV equivalent width variations, it is expected that

$i+\beta>90^{\circ}$.

Indeed, we obtain $i+\beta=106 \pm 28^{\circ}$.

\subsection{Model}

With the known angles $i$ and $\beta$ in the oblique dipolar model, the strength of the magnetic field at the poles, i.e the maximum field, can be determined.

The oblique dipolar rotator model we used was described by Stift (1975). Starting with the observer's system where the $z$-axis is defined by the line-of-sight, a surface point on the visible hemisphere of the star is given by a vector. This vector is transformed into the corotating system of the star, followed by a rotation around the rotational $z$-axis. The position of the dipole system relative to the rotational system is uniquely described by three Eulerian angles. The effect of the three Eulerian rotations can be summarized without loss of generality by a rotation with $\beta$ around the $x$-axis. Given the offset coordinates of the dipole in the rotation system, we obtain the coordinates of the surface point relative to the dipole system and the field strength in the dipole system. From this we get the field strength in the observer's system.

To reproduce the measured longitudinal field values, we use a limb darkening law as described in Stift (1975), with $k=$ 0.4 as established by Claret (2000) for this kind of star in the optical. With $i=71^{\circ}$ derived in Sect. 4.2 and $\beta=35^{\circ}$ derived in Sect. 6.2, we model a centered oblique magnetic rotator and fit it to the longitudinal magnetic field data. The best fit is obtained with $B_{0}=19 \pm 15 \mathrm{G}, B_{1}=39 \pm 32 \mathrm{G}$ and a polar field $B_{\text {pol }}=$ $250 \pm 190 \mathrm{G}$, with a reduced $\chi^{2}=1.20$. The values obtained for $B_{0}$ and $B_{1}$ are compatible with the ones obtained from the best sine fit in Sect. 6.1, with a similar $\chi^{2}$ value. Note that the angles $i$ and $\beta$ are anticorrelated, i.e. to keep $B_{0}$ and $B_{1}$ constant when increasing $\beta, i$ and $B_{\text {pol }}$ have to be decreased.

A greyscale representation of the relative contribution of the magnetic dipole to the integrated longitudinal field on the visible hemisphere of the star, at different rotational phases, is shown in Fig. 16. Although the strongest magnetic field is at 

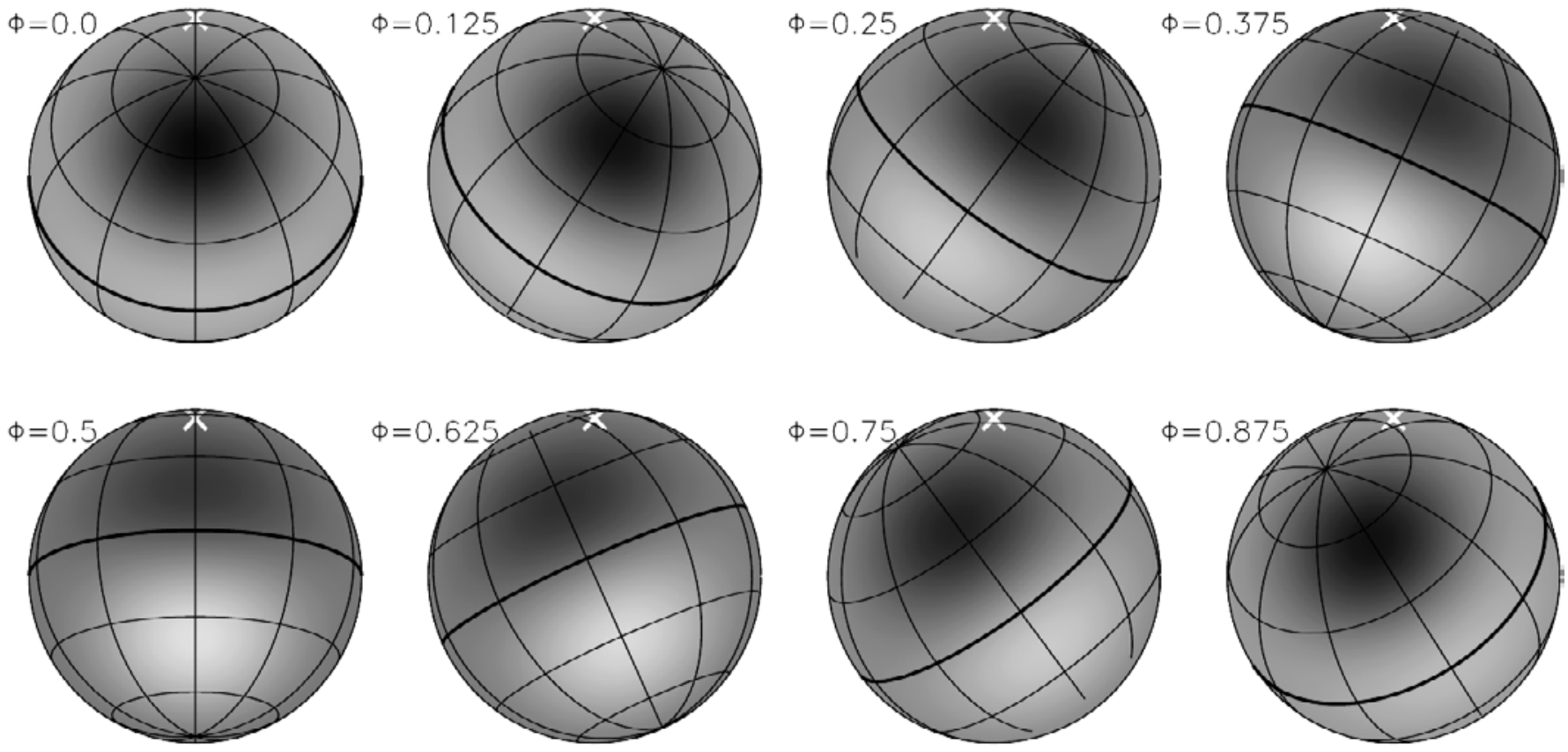

Fig. 16. Greyscale representation of the relative contribution of the magnetic dipole to the integrated longitudinal magnetic field on the visible hemisphere of V $2052 \mathrm{Oph}$, at different rotational phases with step of $\delta \Phi=0.125$. The black color corresponds to positive field values and the white color to negative field values. The phase runs from the top left panel to the lower right panel and corresponds to the convention used in Fig. 15. A grid of magnetic longitudes and latitudes is overplotted, with the magnetic equator shown as a thicker black line. The rotation axis is shown with a white cross. Although the strongest magnetic field is at the magnetic poles, the positions on the stellar surface that contribute the most to the longitudinal field are not at the poles, due to a geometrical effect and to the limb darkening effect.

the magnetic poles, the positions on the stellar surface that contribute the most to the longitudinal field are not at the poles, due to a geometrical effect and to the limb darkening effect.

\section{Conclusions and discussion}

\subsection{Rotation}

We determined the $3.638833 \mathrm{~d}$ stellar rotation period from the variations in equivalent width of the UV stellar wind lines. We found that the minimum intensity of optical photospheric lines (He I and Si III lines) also varies with the rotation period. The strength of the absorption in the UV resonance lines varies in phase with the optical Si III line, but in antiphase with the optical He I lines: when the highly ionized lines are deep, the He I lines are weak, and vice versa. The equivalent widths of the Si III lines increase with higher temperature for stars with $T_{\text {eff }}<27000 \mathrm{~K}$ (Becker \& Butler 1990). The equivalent widths of the He I lines vary in the opposite way for stars with $T_{\text {eff }}>21000 \mathrm{~K}$ (Leone \& Lanzafame 1998). Thus the behaviour observed in V2052 Oph could be easily explained by temperature variations.

In the context of a magnetic star, these variations could also be explained by a temperature difference between the magnetic poles and the rest of the stellar surface. The deepest profile of the He I line corresponds to the minimum temperature. We can estimate the temperature variations from the visual magnitude changes measured by Hipparcos. The frequency detected by Hipparcos (Perryman et al. 1997) corresponds to $f_{1}$ (radial mode, see Sect. 7.2). However, an analysis of the Hipparcos data with the LS method shows that the second detected frequency is $f_{3}$, i.e. twice the rotational frequency. It is therefore possible that the star undergoes temperature variations due to its radial mode, as was already found by Morton \& Hansen (1974), plus apparent temperature variations due to the magnetic poles passing through the line of sight of the observer.

However, it appears that the amplitude of these additional temperature variations needed to explain the rotationally modulated line depths of V 2052 Oph is of the same order as the one produced by the pulsations, i.e. about $2000 \mathrm{~K}$, which is higher than the temperature variation observed for Ap stars (e.g. $600 \mathrm{~K}$ for 41 Tau, Sokolov 1999) with a ten times stronger field than the one of V $2052 \mathrm{Oph}$. We conclude that the weak magnetic field of V 2052 Oph is unlikely to produce such strong additional temperature effects and that it is mainly an abundance effect (see Sect. 7.4).

\subsection{Pulsations}

We confirm that $\mathrm{V} 2052 \mathrm{Oph}$ pulsates radially with $f_{1}=$ $7.15 \mathrm{c} \mathrm{d}^{-1}$. This powerful frequency is detected in all studied lines in the lpv, radial velocity and minimum intensity measurements. We found that the star also hosts a non-radial pulsation mode with $f_{2}=6.82 \mathrm{c} \mathrm{d}^{-1}$ and $l=3$ or 4 , detected in the lpv. This is the first detection of multiperiodicity in this star. A frequency $f_{3}=0.55 \mathrm{c} \mathrm{d}^{-1}$ is detected in the lpv, radial velocity and minimum intensity measurements. This corresponds to twice the rotational frequency.

In the case of Ap and roAp magnetic oblique rotators, the oblique pulsator model also applies (Kurtz 1982), i.e. the 
pulsation axis is aligned with the magnetic axis, being oblique to the rotation axis. As the star rotates, the angle between the observer and the pulsational axis changes. Therefore one expects to see rotational modulation of the amplitude and phase of oscillations. This seems to also be the case in V 2052 Oph (see Fig. 13).

\subsection{Magnetic field}

Although the $S / N$ of the direct measurements of the longitudinal component of the magnetic field of V 2052 Oph is weak due to the faintness of the star, a magnetic field is likely detected by combining all measurements and folding them with the known rotation period. The angles derived for the oblique dipole, $i=71 \pm 10^{\circ}$ and $\beta=35 \pm 18^{\circ}$ are such that the magnetic poles pass through the observer's line of sight. This is confirmed by the detection of $f_{3}$ in the optical photospheric lines, with one pole appearing stronger than the other one, as expected from the difference in strength of the two absorption minima observed in the lines. The dipole model gives then $B_{\text {pol }}=250 \pm 190 \mathrm{G}$.

Other evidence for the presence of a magnetic field in this star is the fact that the extremum field occurs within the uncertainty limits at the same epoch as the predicted mimimum $E W$ of the stellar wind lines, i.e. identical to what is observed in other magnetic stars, such as $\beta$ Cep.

\subsection{Abundance}

Our analysis shows that the star is chemically peculiar: it is He-strong and O-weak. It is known that the oxygen abundance in a star forming region is often $3 / 4$ of the solar abundance. However, the oxygen in V 2052 Oph is even less abundant than that fraction and therefore cannot be attributed to an underabundance in the star forming region. In addition, Smith \& Groote (2001) found that V 2052 Oph has a low metal abundance.

Peculiar chemical compositions are usually found in magnetic stars, in which microscopic diffusion effects allow elements with a high atomic mass to sink in the atmosphere under the dominant influence of gravity, while elements which can absorb photons of many wavelengths from the outward flow of radiation through the star are lifted towards the surface. The first element with a peculiar abundance is then helium (Michaud et al. 1987). In most of the stars this process is usually cancelled by mixing processes (e.g. convection in cool stars). However, the presence of a magnetic field inhibits mixing motions in the outer layers, and hence produces chemical peculiarities.

The line profile modulation can rather be attributed to inhomogeneous surface distribution of chemical abundances. This effect is well-known in Ap stars. Michaud et al. (1987) showed that in the presence of mass loss, the He abundances in the line-forming depths may be modified by chemical separation that takes place not only in the atmosphere but also in the wind and in the envelope of the star. At $T_{\text {eff }}=25000 \mathrm{~K}$ this leads to differential winds. Groote \& Hunger (1997) found that the magnetic He-strong B star $\sigma$ Ori E, which presents many similarities with V $2052 \mathrm{Oph}$, has surface patches of He-enrichment related to fractionation of the wind. Smith \& Groote (2001) extended this model by proposing the presence of co-rotating torus-shaped clouds between the magnetic poles and equator. Hunger \& Groote (1999) found that He-fractionation occurs for stars with $15500 \mathrm{~K}<T_{\text {eff }}<30000 \mathrm{~K}$. They also stated that a fraction of hydrogen or helium is forced to fall back to the star by gravity, because it was not yet coupled at the critical point or because it did not attain the escape velocity and was decoupled further out. If a magnetic field is present this reaccreation proceeds along the same trajectories as those of the ascending particles. Thus the abundance anomalies should show up at the wind bases, i.e the magnetic poles. They conclude that all He-strong main sequence stars are also magnetic stars, otherwise the turbulence would suppress the inhomogeneities.

The influence of the inhomogeneous abundance distribution on the shape of the line profiles could be studied in more detail with Doppler imaging techniques.

\subsection{Comparison with other pulsating magnetic $B$ stars}

V 2052 Oph is very similar to $\beta$ Cep, which hosts a magnetic field. The parameters are very similar, except that $\beta$ Cep has its magnetic axis almost perpendicular to its rotational axis $\left(\beta=85^{\circ}\right)$, whereas for $\mathrm{V} 2052 \mathrm{Oph} \beta=35^{\circ} . \beta \mathrm{Cep}$ has a magnetically confined wind (Donati et al. 2001), i.e. the stellarwind streams from both magnetic hemispheres collide with each other in the magnetic equatorial plane, producing a strong shock, an extended post-shock region and a high-density cooling disc (for more details see Babel \& Montmerle 1997). This is consistent with the magnetic measurements and wind UV data, i.e. $B_{\text {long }}=0$ when the $\mathrm{UV}$ absorption is at maximum. We find that the same model would apply for V 2052 Oph (see Fig. 15), with the same agreement in phase, i.e. the wind is magnetically confined. The magnetic field strength of the two stars and their wind are very similar.

Moreover $\beta$ Cep pulsates radially and non-radially, similar to V 2052 Oph. From ROSAT observations (Berghoefer et al. 1996) a non-detection, i.e. an upper limit, of X-rays was obtained with $\log L_{X} \leq 30.22$. Using the luminosity determined in this paper leads to $\log \left(L_{\mathrm{X}} / L_{\mathrm{bol}}\right) \leq-7.17$ for $\mathrm{V} 2052 \mathrm{Oph}$, which is similar to the detection with $\log \left(L_{\mathrm{X}} / L_{\mathrm{bol}}\right)=-7.16$ obtained for the nearer star $\beta$ Cep. This is consistent with both stars having the same intrinsic X-ray emission. Similarly, if V 2052 Oph would have the same IR excess as $\beta$ Cep the lack of detection of an IR excess from IRAS data is compatible with the difference in distance (A. Lenorzer, private communication). Thus, from all these aspects, these two stars resemble each other.

However, the slowly rotating Be star $\beta$ Cep sometimes exhibits $\mathrm{H} \alpha$ line emission (Kaper \& Mathias 1995; Neiner et al. 2001). The $\mathrm{H} \alpha$ line of $\mathrm{V} 2052 \mathrm{Oph}$ has never been observed in emission, and this star is therefore not classified as a Be star, but given the many specific similarities, it is tempting to suggest a $\mathrm{H} \alpha$ line-emission phase in the future. 
$\mathrm{V} 2052 \mathrm{Oph}$ is the second discovered magnetic pulsating B star, after $\beta$ Cep. Such stars are apparently rather rare, but will provide the most massive examples of stars useful for asteroseismological (and hence evolutionary) tests. We hope to be able to confirm the direct detection of a magnetic field in V $2052 \mathrm{Oph}$, and other similar stars, and analyze the rotational modulation of the field, using the new spectropolarimeter ESPaDOnS, which will be installed at CFHT in January 2004 and is expected to be at least 10 times more efficient than Musicos.

Acknowledgements. We are grateful to J. Cami, P. Ehrenfreud, L. Kaper and A. van den Meer for providing the VLT spectrum and J. Jimenez for reducing it. $\mathrm{CN}$ wish to thank J.-F. Donati and E. Verdugo for useful discussions concerning the TBL fringe correction, S. Solanki for his help on the dipole model and A. de Koter for interesting discussions on winds. Thanks also goes to E. Verdugo for her contribution to the determination of the rotation period and to the referee D. Gies.

\section{References}

Andrievsky, S. M., Korotin, S. A., Luck, R. E., \& Kostynchuk, L. Y. 1999, A\&A, 350, 598

Babel, J., \& Montmerle, T. 1997, A\&A, 323, 121

Becker, S. R., \& Butler, K. 1990, A\&A, 235, 326

Beeckmans, F., \& Burger, M. 1977, A\&A, 61, 815

Berghoefer, T. W., Schmitt, J. H. M. M., \& Cassinelli, J. P. 1996, A\&AS, 118,481

Bohlin, R. C., Henrichs, H. F., \& Nichols, J. S. 1994, A\&AS, 105, 305

Cardelli, J. A., Clayton, G. C., \& Mathis, J. S. 1989, ApJ, 345, 245

Claret, A. 2000, A\&A, 363, 1081

Cugier, H., Dziembowski, W. A., \& Pamyatnykh, A. A. 1994, A\&A, 291, 143

Diplas, A., \& Savage, B. D. 1994, ApJS, 93, 211

Domiciano de Souza, A., Janot-Pacheco, E., Leister, N. V., et al. 2000, in The Be Phenomenon in Early-Type Stars, IAU Coll. 175, ed. M. A. Smith, H. F. Henrichs, \& J. Fabregat, ASP Conf. Ser., 214, 276

Domiciano de Souza Jr., A. 1999, Master's Thesis, IAG-USP, Brazil

Donati, J.-F., Semel, M., Carter, B. D., Rees, D. E., \& Collier Cameron, A. 1997, MNRAS, 291, 658

Donati, J.-F., Catala, C., Wade, G. A., et al. 1999, A\&AS, 134, 149

Donati, J.-F., Wade, G. A., Babel, J., et al. 2001, MNRAS, 326, 1265

Emilio, M. 1997, Master's Thesis, IAG-USP, Brazil

Foster, G. 1995, AJ, 109, 1889

Foster, G. 1996, AJ, 111, 541

Gray, D. F. 1976, The observation and analysis of stellar photospheres (Wiley-Interscience)
Grevesse, N., \& Sauval, A. J. 1998, Space Sci. Rev., 85, 161

Groote, D., \& Hunger, K. 1997, A\&A, 319, 250

Henrichs, H. F., Kaper, L., \& Nichols, J. S. 1994, A\&A, 285, 565

Henrichs, H. F., de Jong, J. A., \& Kaper, L. 1998, in UV Astrophysics Beyond the IUE Final Archive, ESA-SP 413, 157

Henrichs, H. F., de Jong, J. A., Donati, J.-F., et al. 2000, in The Be Phenomenon in Early-Type Stars, IAU Coll. 175, ed. M. A. Smith, H. F. Henrichs, \& J. Fabregat, ASP Conf. Ser., 214, 324

Henrichs, H. F., de Jong, J., Donati, J. F., et al. 2003, A\&A, to be submitted

Heynderickx, D., Waelkens, C., \& Smeyers, P. 1994, A\&AS, 105, 447

Hubeny, I., \& Lanz, T. 1995, ApJ, 439, 875

Hunger, K., \& Groote, D. 1999, A\&A, 351, 554

Jerzykiewicz, M. 1972, PASP, 84, 718

Kaper, L., \& Mathias, P. 1995, in Astrophysical Applications of Stellar Pulsation, held in Cape Town in 1995, IAU Colloq. 155, ed. R. S. Stobie, \& P. A. Whitelock, ASP Conf. Ser., 83, 295

Kurtz, D. W. 1982, MNRAS, 200, 807

Leone, F., \& Lanzafame, A. C. 1998, A\&A, 330, 306

Michaud, G., Dupuis, J., Fontaine, G., \& Montmerle, T. 1987, ApJ, 322, 302

Morton, A. E., \& Hansen, H. K. 1974, PASP, 86, 943

Neiner, C., Henrichs, H. F., Geers, V. C., \& Donati, J.-F. 2001, IAU Circ., 7651, 3

Neiner, C., Hubert, A.-M., Floquet, M., et al. 2002, A\&A, 388, 899

Neiner, C., Geers, V. C., Henrichs, H. F., et al. 2003, A\&A, 406, 1019

Papaj, J., Wegner, W., \& Krelowski, J. 1990, MNRAS, 246, 408

Perryman, M. A. C., Lindegren, L., Kovalevsky, J., et al. 1997, A\&A, 323, L49

Pike, C. D. 1974, PASP, 86, 681

Rountree, J., \& Sonneborn, G. 1991, ApJ, 369, 515

Rountree Lesh, J. 1968, ApJS, 17, 371

Schaller, G., Schaerer, D., Meynet, G., \& Maeder, A. 1992, A\&AS, 96, 269

Schrijvers, C., Telting, J. H., Aerts, C., Ruymaekers, E., \& Henrichs, H. F. 1997, A\&AS, 121, 343

Shore, S. N. 1987, AJ, 94, 731

Smith, M. A., \& Groote, D. 2001, A\&A, 372, 208

Sokolov, N. A. 1999, Informational Bulletin on Variable Stars, 4781, 1

Sonneborn, G., Garhart, M. P., \& A., G. C. 1987, in Physics of Be Stars, held in Boulder in 1986, ed. A. Slettebak, \& T. P. Snow (Cambridge University Press), IAU Colloq., 92, 286

Stibbs, D. W. N. 1950, MNRAS, 110, 395

Stift, M. J. 1975, MNRAS, 172, 133

Wolff, S. C., Edwards, S., \& Preston, G. W. 1982, ApJ, 252, 322

Wolff, S. C., \& Heasley, J. N. 1985, ApJ, 292, 589

Zboril, M., \& North, P. 2000, Contributions of the Astronomical Observatory Skalnate Pleso, 30, 12 
C. Neiner et al.: Rotation, pulsations and magnetic field in V 2052 Oph, Online Material p 1

\section{Online Material}


C. Neiner et al.: Rotation, pulsations and magnetic field in V 2052 Oph, Online Material p 2

Table 7. Table of longitudinal magnetic field measurements. Columns 1,2 and 3 indicate the number, HJD and phase (with $P=3.638833 \mathrm{~d}$ ) of each measurement respectively. Columns 4 and 5 give the longitudinal field measurement and its error bar. Columns 6 and 7 give the null polarisation and its error bar.

\begin{tabular}{|c|c|c|c|c|c|c|c|c|c|c|c|c|c|c|}
\hline \# & JD & $\mathrm{Ph}$ & $\sigma B$ & $N \sigma N$ & $\#$ & HJD & $\mathrm{Ph}$ & $B \sigma B$ & $N \sigma N$ & \# & HJD & h & $B \sigma B$ & $N \sigma N$ \\
\hline 1 & 0.43 & 0.113 & 354134 & 117128 & 0 & 355. & 60.707 & 81221 & 66219 & 77 & 361. & .320 & -149167 & 67165 \\
\hline 2 & 0.55 & 0.146 & 68140 & -2 & 1 & 355.58 & 80.714 & -309235 & -4 & 78 & 361.4 & 50.327 & -12 & 70 \\
\hline 3 & 5.40 & 0.479 & -333152 & 86148 & 2 & 356 & 40.949 & -3 & -219 & 79 & 361 & .333 & 32167 & 164 \\
\hline 4 & 5.43 & 0.489 & 3144 & -263140 & 43 & 356 & 60.955 & 72167 & -238 & 80 & 361. & 0.339 & -145167 & 165 \\
\hline 5 & 5.48 & 0.500 & -1164 & 28101 & 44 & 356 & 80.961 & 1951 & -282194 & 81 & 361 & 0.345 & 61172 & 17170 \\
\hline 6 & 5.52 & 0.512 & -333200 & 477196 & 45 & 356 & 10.968 & 19184 & -61182 & 82 & 364.37 & 70.130 & 110224 & -245224 \\
\hline 7 & 5.56 & 0.522 & -475206 & 202203 & 46 & 356 & 0.974 & 244193 & -145 & 83 & 364 & 0.136 & -15 & 75252 \\
\hline 8 & 6.40 & 0.752 & -102190 & -150 & 47 & 356 & 0.980 & -298198 & 159194 & 84 & 364 & 42 & 4247 & 140246 \\
\hline 9 & 6.43 & 0.762 & 23020 & -102 & 48 & 356 & 70.986 & -108 & -80 & 85 & 364 & .149 & 217218 & 128217 \\
\hline 10 & 6.47 & 0.773 & 214210 & 208 & 9 & 3 & 221 & 42167 & -23164 & 86 & 364 & 5 & -4872 & 264 \\
\hline 11 & 6.51 & 0.783 & 176248 & -272248 & 50 & 357 & 0.227 & -57176 & 11171 & 87 & 364. & .161 & -246294 & 291 \\
\hline 12 & 6.54 & 0.793 & -457355 & 174355 & 51 & 357 & 234 & 226172 & -9 & 88 & 36 & 8 & -10 & 293 \\
\hline 13 & 6.59 & 0.805 & 204296 & -1 & 52 & 3 & 10 & 609170 & 107 & 89 & 36 & 2 & -9 & 190 \\
\hline 14 & 10.38 & 0.847 & 115218 & 48216 & 53 & 35 & 20.246 & 313167 & 20165 & 90 & 365 & .409 & -172183 & -263182 \\
\hline 15 & 10.41 & 0.857 & 550221 & -64218 & 54 & 357 & 40.251 & 68173 & 9 & 91 & 365 & 15 & 162186 & -77182 \\
\hline 16 & 10.45 & 0.867 & -437195 & 3193 & 55 & 358 & 0.483 & -492 & -22 & 92 & 365 & 422 & 60184 & 40180 \\
\hline 17 & 13.47 & 0.697 & 13138 & -241 & 56 & 358 & 00 & s & 40 & 93 & 365 & 428 & -46200 & 147197 \\
\hline 18 & 13.50 & 0.706 & -38142 & -13 & 57 & 358. & 0.495 & -556229 & 28 & 94 & 365 & 35 & -60188 & 187 \\
\hline 19 & 13.55 & 0.718 & 94174 & -171175 & 58 & 358. & 50.502 & -139206 & -284204 & 95 & 365 & .441 & -389191 & 193186 \\
\hline 20 & 13.59 & 0.729 & 7422 & 163 & 59 & 3 & 3 & -19622 & 380 & 06 & I & ( & -33 & 221 \\
\hline 21 & 17.38 & 0.771 & 7240 & 69237 & 60 & 358. & 10.518 & -271204 & -109200 & 97 & 366.3 & 90.684 & -404 & 318259 \\
\hline 22 & 17.44 & 0.787 & 5119 & -104191 & 61 & 358 & 40.525 & -163223 & 7222 & 98 & 366 & .691 & 40216 & 227215 \\
\hline 23 & 17.48 & 0.798 & -323 & 17 & 62 & 35 & 70.755 & 01220 & 256230 & נק & $\pi$ & 0 & -3 & 244 \\
\hline 24 & 17.52 & 0.808 & -411208 & -37202 & 63 & 359 & 90.761 & -36196 & -15 & 100 & 366. & 704 & -15 & 218260 \\
\hline 25 & 17.55 & 0.818 & -272216 & 882 & 64 & 35 & 07 & 239192 & -17 & 101 & 36 & 0.711 & 39 & 263 \\
\hline 26 & 350.4 & 0.301 & 1194436 & 559437 & 65 & 359 & 30 & 1282 & -11 & 102 & 36 & 54 & 63 & 340262 \\
\hline 27 & 350.4 & 0.308 & -380375 & 170373 & 66 & 359 & 86 & 42816 & 3 & 103 & 36 & 60 & 168282 & 76280 \\
\hline 29 & 350.52 & 0.322 & -89289 & 177287 & 67 & 359.5 & 10.793 & 199189 & -1711 & 104 & 367 & 20.967 & 87264 & 96262 \\
\hline 30 & 350.54 & 0.329 & -374299 & 217294 & 68 & 359. & 30.799 & $44186-$ & -131183 & 105 & 367. & 0.973 & -177235 & -50229 \\
\hline 31 & 350.5 & 032 & 0313 & 206312 & 9 & 360 & .035 & -79266 & 8261 & 106 & & & 172 & 98 \\
\hline 32 & 351 & 0.598 & -390290 & 330290 & 70 & 360. & 10.041 & 263242 & -76240 & 107 & 367 & .986 & -40262 & 250265 \\
\hline 33 & 351.5 & 0.604 & -234272 & 126273 & 71 & 360 & 0.047 & 18226 & 227226 & 108 & 367 & 0.993 & 122219 & 6217 \\
\hline 34 & 35 & 0.168 & 21227 & -211228 & 72 & 360 & 60.0 & 289242 & 652238 & 109 & 368 & r & -2122 & 21211 \\
\hline 35 & 353.6 & 0.174 & -79244 & 114243 & 73 & 360. & 80.059 & -140224 & 8222 & 110 & 368. & 0.249 & 40197 & 12198 \\
\hline 36 & 353.64 & 0.180 & -312402 & -406402 & 74 & 360. & 0.065 & -185234 & 133231 & 111 & 368. & 0.255 & -10168 & -103164 \\
\hline 37 & 355.4 & 0.686 & -398257 & 204255 & 75 & 361 & 80.308 & -5184 & -252181 & 112 & 368 & .261 & -332185 & 259184 \\
\hline 38 & 355.5 & 0.692 & -81239 & -592236 & 76 & 361. & 10.314 & 171170 & -135166 & 113 & 368.5 & 10.268 & -195231 & 225228 \\
\hline 39 & 355.53 & 0.700 & -172225 & 112222 & & & & & & & & & & \\
\hline
\end{tabular}

